\title{
Validating Basic Surface Variables in the Australian Bureau of Meteorology Model with CEOP EOP3 In-situ Data
}

\author{
Lawrie RIKUS \\ Bureau of Meteorology Research Centre, Melbourne, Australia \\ (Manuscript received 21 February 2006, in final form 8 September 2006)
}

\begin{abstract}
The Coordinated Enhanced Observing Period (CEOP) Project has initiated the collection of model output location time series (MOLTS) data from numerical weather prediction and assimilation centers, including the Australian Bureau of Meteorology. These were designed to complement the collection of insitu observational data sets at the same 41 locations. This study is a preliminary attempt to assess the differences and similarities between the MOLTS and time series of the in-situ data. The MOLTS from the Bureau's global model for a number of atmospheric surface variables are compared with in-situ data for 6 locations for the entire year of EOP3 (October 2002 to September 2003). Using surface pressure as a prototype a number of preliminary comparisons of different properties of the two types of data are performed. The model fields from the 6 hour assimilation forecast series and from the concatenated 12 to 36 hour forecasts are first compared with hourly in-situ observations in simple gross terms; simple statistics and scatter plots have been obtained to identify areas for further investigation. Global wavelet power spectra are compared to show the behavior of the dominant modes of variability and the average annual diurnal variations of the variables are derived. Some case studies with other variables with increasing sensitivity to sub-grid scales and increasing dependency on surface properties are then documented. The model and in-situ time series show similar behavior for surface pressure with greater differences for the other variables. Precipitation shows the greatest differences.
\end{abstract}

\section{Introduction}

\subsection{CEOP and MOLTS}

The Coordinated Enhanced Observing Period (CEOP) Project was first proposed at the Global Energy and Water Cycle Experiment (GEWEX) Hydrometeorology Panel (GHP) in 1997 with an Implementation Plan for what has become designated as Phase 1 defined in May 2001. The Implementation Plan defined a primary focus for the collection of satellite, in-situ and numerical weather prediction data for the Enhanced Observing Period 3 (EOP3), from $1^{\text {st }}$ October 2002 to $30^{\text {th }}$ September 2003 with the

Corresponding author: Lawrie Rikus, Bureau of Meteorology Research Centre, G.P.O. Box 1289, Melbourne, Vic, 3000, Australia.

Email: l.rikus@bom.gov.au

(C) 2007, Meteorological Society of Japan following year designated as EOP4. Planning documents and various reports on the project are available from the CEOP website (http:// www.ceop.net/). The basic underlying task of CEOP is to establish a unified collection of large, high quality datasets of satellite, in-situ and numerical weather prediction (NWP) data designed to focus on diagnosing the energy and water cycles over continental scale regions from diurnal to annual time scales. As part of that process three main data centers have been established to collect and disseminate data along with data mining studies to improve access and increase the utility of the data.

A number of NWP and data assimilation centers have contributed a combination of gridded data and Model Output Location Time series (MOLTS) data to the project. The latter are time series of model variables at 41 specified 
locations including GEWEX Continental Scale Experiments, Atmospheric Radiation Measurement (ARM) sites and the Murray-Darling Basin (MDB). These reference sites have hydrological and basic weather data available over the entire EOP3 with a temporal resolution of a half hour, which is available in four standard ASCII formats covering surface meteorological and radiation data (SFC), flux data (FLX), soil temperature and moisture data (SLM) and meteorological tower data (TWR). It is available from the data management site at http://www .eol.ucar.edu/projects/ceop/dm/.

\subsection{The Bureau of Meteorology Global Numerical Prediction Model}

The Australian Bureau of Meteorology is one of the operational NWP centers contributing to the CEOP dataset. The version of the model used to generate the CEOP MOLTS data was that in operation during EOP3. At that time the assimilation system was a multi-variate statistical interpolation scheme (Seaman et al. 1995) with a 6 hour cycle. In this system data is inserted into first guess fields which are 6 hour model forecasts from the previous analysis to produce the next analysis. Moisture is assimilated independently within this 6 hour cycle in a univariate scheme. The relevant version of the operational medium range prediction model (GASP) is a global spectral model with a horizontal resolution of T239 (equivalent to about a $75 \mathrm{~km}$ east-west grid) and 29 vertical (sigma ${ }^{1}$ ) levels with the top level at about $10 \mathrm{hPa}$ and the lowest level at about $990 \mathrm{hPa}$. Prognostic variables are surface pressure, temperature, water vapor mixing ratio, vorticity and divergence.

Radiative transfer is parameterized by a twoband Lacis-Hansen (1974) scheme for shortwave and a Fels-Schwarzkopf scheme for the longwave (Fels and Schwarzkopf 1975; Schwarzkopf and Fels 1991). There are no aerosols or trace gases included. The convection scheme is a slightly modified version of the original mass flux scheme (Tiedke 1989) coupled to a large scale condensation scheme which instantaneously rains out grid scale supersaturation. The large scale cloud fraction is diagnosed

1 Sigma levels are defined as pressure divided by surface pressure. from the relative humidity with additional stability dependent low cloud and is restricted to 3 non-contiguous layers (Rikus 1991). Cloud condensate content is parameterized diagnostically using temperature as the predictor (Lemus et al. 1997). The identification of ice or water is also a smooth function of temperature ranging from completely water down to $0^{\circ} \mathrm{C}$ to completely ice at $-20^{\circ} \mathrm{C}$.

The surface turbulent eddy fluxes of heat, moisture and momentum are parameterized with a Monin-Obhukov scheme with stability dependent coefficients determined as suggested by Louis (1979). The vertical diffusion is applied generally in the lower half of the atmosphere and for any unstable layers. Prognostic soil moisture is modeled by an interactive scheme which uses a fixed "bucket" with a with uniform field capacity of $0.15 \mathrm{~m}$ (Manabe and Holloway 1975) and source terms due to precipitation, evaporation and melting snow. Soil temperature is computed from an energy balance at the surface using the heat storage in two layers underpinned by a climatologically defined deep soil layer. Surface roughness lengths over land and sea-ice are prescribed constants which take no account of vegetation type etc. Over the oceans the roughness length is determined from the surface wind stress (Charnock 1955). The land albedo is from climatology (Hummel and Reck 1979), and is solar zenith angle dependent over the ocean (Payne 1972). Sea surface temperatures were specified by the operational Bureau analysis scheme (Smith 1995) and updated regularly every two weeks.

\subsection{MOLTS from the Bureau's global assimilation system}

The CEOP plans called for two basic MOLTS; output from the assimilation cycle and equivalent data from the last 24 hours of 36 hour forecasts. So far only the MOLTS data for EOP3 are available from the Bureau's model. It was generated by running six hourly forecasts based on the operational analysis files from the Bureau of Meteorology's global NWP system for the entire year of EOP3 with hourly output of the closest model columns to each of the 41 designated reference locations. The individual analysis and forecast files were concatenated together to form two separate contiguous time series for EOP3 with a temporal 
Table 1. Comparison of the chosen in-situ sites and the corresponding BMRC model MOLTS points.

\begin{tabular}{|l|c|c|c|c|c|c|c|}
\hline \multicolumn{2}{|c|}{} & \multicolumn{3}{|c|}{ in-situ } & \multicolumn{3}{c|}{ MODEL } \\
\hline Name & Surface & $\begin{array}{c}\text { Latitude } \\
\text { Deg. N }\end{array}$ & $\begin{array}{c}\text { Longitude } \\
\text { Deg. E }\end{array}$ & $\begin{array}{c}\text { Elevation } \\
\text { meters }\end{array}$ & $\begin{array}{c}\text { Latitude } \\
\text { Deg. N }\end{array}$ & $\begin{array}{c}\text { Longitude } \\
\text { Deg. E }\end{array}$ & $\begin{array}{c}\text { Elevation } \\
\text { meters }\end{array}$ \\
\hline Lindenberg & land & 52.17 & 14.12 & 73 & 52.02 & 13.20 & 77 \\
\hline SGP & land & 36.61 & -97.49 & 313 & 36.30 & -98.10 & 379 \\
\hline WPO & sea & 7.04 & 134.27 & 2 & 7.11 & 134.25 & -8 \\
\hline Equatorial Island & land & -0.20 & 100.32 & 699 & -0.37 & 99.75 & 90 \\
\hline MDB & land & -35.66 & 148.15 & $1200 *$ & -35.55 & 147.60 & 473 \\
\hline Darwin & land & -12.43 & 130.89 & 30 & -12.35 & 130.50 & 19 \\
\hline Ringwood (SGP) & land & 36.43 & -98.28 & 418 & 36.30 & -98.10 & 379 \\
\hline Kyeamba (MDB) & land & -35.43 & 147.20 & 212 & -35.55 & 147.60 & 473 \\
\hline
\end{tabular}

* Note that the CEOP reference site for the MDB is based around Tumbarumba, a mountain site which in fact does not form part of the data set. A more representative height is probably about $200 \mathrm{~m}$.

resolution of one hour. The first, labeled as ASSIM, is the concatenation of hourly output from the 6 hour forecasts connecting the archived operational analysis to the next analysis, with the last forecast equivalent to the first guess. A second (FORC) series was formed from concatenated 12 to 36 hour forecasts started at 12 UTC each day.

Global assimilation systems and models are designed to analyze and simulate the characteristics of meteorological fields at large scales and in principle are unable to provide information on how the distribution and values of those fields vary on sub-grid scales. Station data, on the other hand, is susceptible to weather phenomena which are small relative to the grid scale of the model and which can cause it to differ substantially from the grid scale. This paper attempts to assess some aspects of these differences.

\section{Comparing MOLTS and in-situ data}

\subsection{Choice of sites}

The CEOP specification suggests 41 locations for MOLTS output, but not all of these have insitu data for the entire EOP3 period and none have the complete set of requested variables. As a pilot study to assess the difference in character between the MOLTS and in-situ data this paper considers a selected subset with as complete as possible data coverage of a common set of variables for the EOP3 period. The details of these are given in Table 1 .

a) The ARM Southern Great Plains (SGP) location in north-central Oklahoma, USA, (http://www.arm.gov/sites/sgp.stm) was chosen because it has a comprehensive set of observational data and multiple measuring stations covering a large 'grid size' region embedded in a data rich part of the globe. The closest station to the model's MOLTS point was Ringwood (Fig. 1).

b) The Lindenberg location in Northern Germany (Neisser et al. 2002; Beyrich et al. 2002) was chosen as a representative of mid-high latitudes; of the two stations the Falkenberg station was used because it had data for the entire period.

c) The Darwin site from the ARM Tropical Western Pacific dataset (http://www.arm .gov/sites/twp.stm) was chosen as a good representative for convectively dominated weather regimes with monsoon seasonality.

d) The Murray Darling Basin (MDB) data set (Siriwardena et al. 2003) which covers a large fraction of south-eastern Australia was chosen because of its comprehensiveness and because it is of particular interest to Australia. The closest station to the model's MOLTS point was Kyeamba (Fig. 1).

e) The Equatorial Island (Kototabang) site in West Sumatra (Murata et al. 2002) was se- 

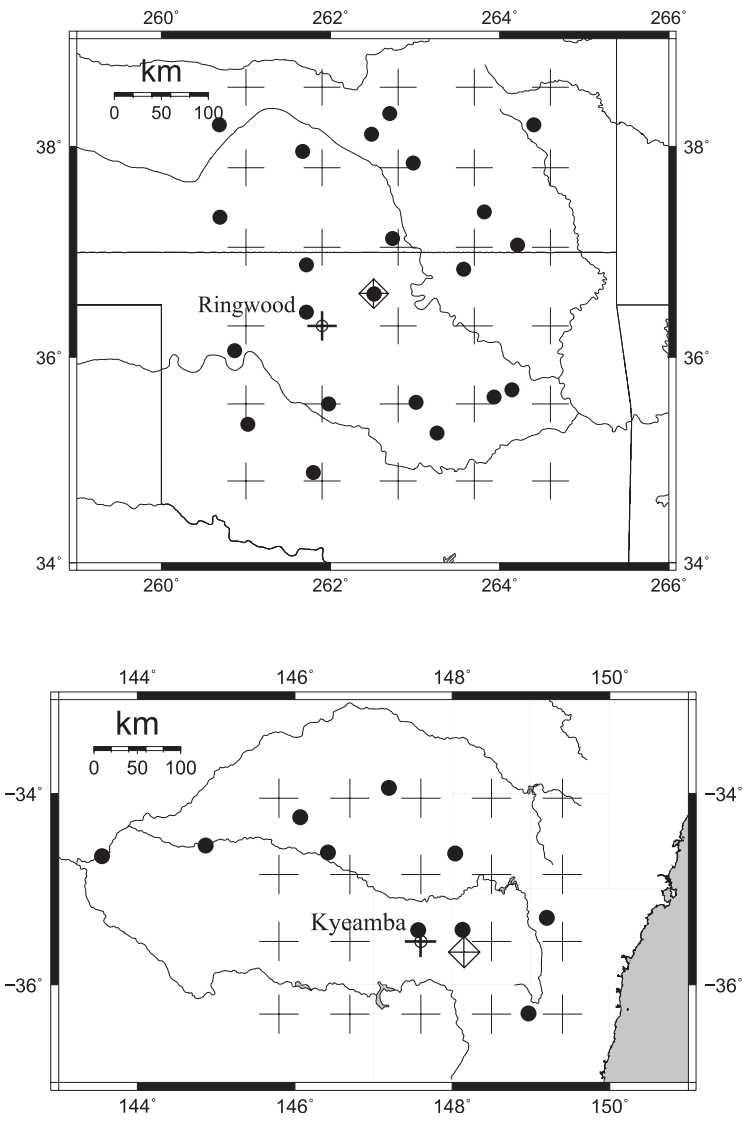

Fig. 1. The distribution of stations in the ARM Southern Great Plains site (upper) and the Murray-Darling Basin (lower). The black spots denote data collection stations, the cross with its center circled is the center of the model MOLTS point with the neighboring grid point centers denoted by crosses. The CEOP defined MOLTS point is denoted by the diamond. The station closest to the model MOLTS point is named.

lected because it is a tropical land site with different characteristics to Darwin. The insitu observation site is some distance from the coast on a plateau. The closest model point is about $50 \mathrm{~km}$ to the west and is actually on the coast and some $600 \mathrm{~m}$ lower in elevation. This mismatch implies that the model forecasts and the observations should be very different as they are sited in different weather regimes and should pro- vide a useful guideline of how important this type of mismatch is for time series comparison.

f) The Western Pacific Ocean (WPO) location (Kubota et al. 2002) was chosen as it contains the only nominally oceanic location and is in a data sparse region dominated by satellite data. Of the two stations Peleliu Island was selected because only it had data for most of EOP3. This location might be problematic for model validation; the model topography does not recognize the presence of the island at the site and therefore treats it as an open ocean point. To the extent that the local meteorology is affected by the landsea heterogeneity some fields could be very different.

The SGP location was designed to be representative of the spatial dimension of a typical model grid. To produce data compatible with the model resolution the individual stations need to be aggregated in some way to produce grid scale fields. As this is a preliminary study the simplest technique was chosen; the data were averaged over all locations for each variable where observations were available. The same procedure was followed for the MDB site. As a simple test to gauge the resolution representativeness of the MOLTS data in the analyses which follow, these mean datasets for the SGP and MDB were compared with the data site closest to the model MOLTS point, Ringwood and Kyeamba respectively.

A relevant question to consider is the representativeness of the model grid point and the reference site; do they have the same physical properties such as surface type, vegetation class, topography etc and, if not, how important are the differences? For example, surface pressure has a strong dependence on surface elevation which can probably be ignored to first order. Surface temperature is essentially determined by the energy budget at the surface and this in turn depends on surface albedo, soil capacitance etc. Surface winds will depend on the friction of the vegetation and local small scale orography which will obviously vary from those prescribed for the model at the grid scale. In the two mean grid locations (SGP and MDB) defined above as an average over all included stations, the averaging ignores the non- 
linearities in the height/pressure dependence of the different fields and the local variation of surface properties may introduce confounding factors.

When comparing data sets there can also be problems with the definition of the variables involved e.g., soil moisture can mean different things in different models and can be different in physical character to the in-situ data. In addition different reference site locations have different sets of data making a 'global' comparison problematic. For example the screen level has been defined as 2 or $1.5 \mathrm{~m}$ at different sites and anemometer readings for surface layer winds are measured at a range of heights. In the following comparisons these differences are ignored and therefore contribute in some measure to the magnitude of the diagnosed differences between the two types of time series.

\subsection{Choice of variables}

The choice of variables was constrained by data availability; of the four types of CEOP insitu data set the most complete were the surface variables. The following basic surface variables are considered here; pressure, temperature, specific humidity, wind speed and precipitation.

Surface pressure is probably the best model variable to validate because it is one of the model's prognostic fields with direct connection to input meteorological data and arguably is the best represented in global data collection terms. It is largely independent of sub-grid scale variations of surface properties and should not vary appreciably over small scales if corrected for differences in local elevation. It also exhibits an obvious and reasonably well understood diurnal variation. These properties make it ideal for a preliminary investigation to evaluate methodologies. The next most common variables collected are the temperature and specific humidity at screen height. In the model this has been given as $1.5 \mathrm{~m}$ which agrees with the sensor height at the Equatorial Island and WPO sites. The other sites have their screen sensors at $2 \mathrm{~m}$ but the difference is probably not significant for well mixed boundary layers. Note that the model's lowest sigma level is some distance above this and the screen level (and $10 \mathrm{~m}$ ) variables are extrapolated from the model fields using a stability dependent fitting process inside the Planetary Boundary Layer (PBL) parameterization scheme. Note also that the assimilation scheme is not optimal for analyzing moisture which implies that the specific humidity at the surface should exhibit larger errors than the better characterized temperature and pressure.

The only dynamical field common to both the model and the in-situ data sets is the wind speed. Most sites have wind speed data available at the nominal $10 \mathrm{~m}$ height with the exception of WPO and the Equatorial Island sites which are at $4 \mathrm{~m}$ and $2 \mathrm{~m}$ respectively and were therefore not considered in the comparison. The wind speed should be sensitive to the surface variations on local scales, particularly those attributable to vegetation and topography.

The least well known quantity of this basic set is the precipitation which exhibits extreme variability at all scales and shows intermittent behavior in most of the hourly time series considered here. Thus it provides a strong challenge for techniques developed to deal with stationary and linear time series and probably requires the application of non-linear, intermittent time series techniques which are beyond the scope of this simple study.

\subsection{Processing the data}

The in-situ surface data was all downloaded from the CEOP in-situ data repository on the JOSS web site except for the MDB data (private communication from A. Seed, Bureau of Meteorology). For each site the standard format surface data ASCII file was converted into netCDF format (Rew and Davis 1990) with half-hourly time steps for easier interface to the data analysis programs. The resolution was then degraded to the hourly resolution of the model data by selecting instantaneous values on the hour, summing accumulated variables for each hour and taking the mean where appropriate.

The ARM Darwin data had to be filtered to remove the zero error of $0.1 \mathrm{~mm} \mathrm{hr}^{-1}$ in the precipitation and to remove large unphysical jumps in surface pressure due to the sensor's sensitivity to precipitation. These changes are consistent with the site data documentation at (http://data.eol.ucar.edu/codiac_data/ceop/docs/ Other/Other_TWP_sfc.htm). 
Each of the in-situ datasets had missing values over the period of EOP3. The largest gaps are for the Western Pacific site which doesn't start until 70 days into the period and the Equatorial Island site which is missing 31 days over June and July 2003. To allow the use of automatic statistical operators and to avoid edge artifacts in wavelet transforms it was decided to fill in the missing values for periods less than 4 days in length with simple estimates designed to preserve the local (in time) diurnal signals without introducing too much model dependence. This still left one period of 5.625 days in the WPO precipitation series which was essentially ignored. For all in-situ datasets, single missing hourly data points were estimated by averaging the neighboring points. For missing periods of a day or less the points were estimated by averaging the values for the same time of day from the two neighboring days. For periods of 2 to 4 days the missing values were estimated by linear interpolation between the bounding days at the same phase of the diurnal cycle. This left the missing month in the Equatorial Island data set which was treated by applying the statistical tools and wavelet transforms independently to the regions of complete data on either side. The Western Ocean data was only analyzed from the beginning of available data and so only includes the last 10 months or so of the EOP3 period. The first month of data from Ringwood (SGP) was incomplete and could not be amended by the interpolation techniques and so was removed entirely. Note that the preliminary data set for the MDB location does not contain surface pressure so this data set was omitted from the surface pressure investigation. The only data for which the percentage of filled points exceeded 1\% was for wind speed for Lindenberg which required just under $15 \%$ of the time period to be interpolated.

\subsection{Validation methodology}

The most basic task to obtain a preliminary view of the representativeness of the model data to the observations is to directly compare the time series by calculating the RMS error, bias and correlation coefficient and their individual variances. Only times for which both time series were available were included in the statistics calculations which were performed af- ter the data gap filling described in the previous section. To gain a visual impression of the agreement between the time series, the scatter of model versus in-situ data for each variable and at each site was plotted along with a simple linear regression fit. In virtually all cases the FORC plots were very similar to the ASSIM series plots (apart from slightly more spread) and so are not reproduced here.

MOLTS data is essentially just an isolated single column of the global model and as such presents a number of difficulties when trying to compare it with the reference site data. The most obvious problem is determining the effective resolution of the two datasets. Some reference locations encompass data from a number of sites spanning areas comparable to the model grid scale e.g., Fig. 1 shows the ARM Southern Great Plains (SGP) data collection area which includes 23 stations distributed over 143,000 square kilometers and the MDB dataset which encompasses several model grid points. Most locations have less than a handful of stations and have a much higher effective resolution than the model for most variables. Most participating models have contributed only a single model grid point for each reference site although the Japanese Meteorological Agency has provided an additional dataset which includes the four points around each MOLTS site which will facilitate studies of effective resolution as well as provide an interface to single column models.

There is some evidence (Yang et al. 2005) that the spatial representativeness of in-situ observations is different for different variables which means that to obtain statistically significant results it is necessary to obtain a balance between temporal and spatial averaging which is variable dependent. If a phenomenon is of short time duration it is more likely to be of small spatial scale as well and is unlikely to be captured by the model. Weather patterns of grid scale size or greater which the model should be able to resolve are more likely to be of longer duration. Hence it makes sense to investigate the two types of time series by decomposing them in time-frequency space using a wavelet analysis which would allow the identification of the dominant modes of variability and how they change with time (Torrence and Compo 1998). This ability to localise the contri- 
butions of different frequencies in time is a major advantage of wavelet techniques as opposed to Fourier transforms when considering time series which have properties which may change with time. An estimate of the power spectrum of the time series can be obtained by averaging the squared absolute value of the wavelet transform amplitude over time to produce the global wavelet power spectrum. Alternatively the time variation of the fluctuations in power over a band of frequencies can be obtained by averaging the wavelet power spectrum over the frequency band. Hence an estimate of the time variation of the diurnal signal can be obtained by defining appropriate limits for this process. For this paper the limits were set to include oscillation time periods between 0.3 and 1.2 days and thus to capture the combined diurnal and semi-diurnal signals. Statistical significance is determined by comparing the spectra with that expected with a red noise background. For all wavelet plots the time series were all normalized to unit variance to facilitate the comparisons between model and in-situ data.

One of the primary foci of the second phase of CEOP is the study of diurnal cycles. Consistent, pervasive large scale physical phenomena such as diurnal tides or fields heavily influenced by the daily radiation cycle should be relatively consistent across long time periods. The simplest way to analyse the diurnal part of a time series is to sort the individual time series into hourly time bins representing the diurnal cycle and take the mean of each bin. Seasonality can be accounted for by using departures from the daily mean rather than the raw values. This process assumes that the nondiurnal behaviour is random (and hence will average out to zero) and that the daily mean corrected diurnal behaviour itself is invariant over the total time of the series; an assumption which is certainly not correct for annual series of variables where seasons play an important role but suffices for a preliminary investigation.

When comparing data, certain limitations inherent in the type of data assimilation employed may need to be taken into account. One of these is model physics spin-up; due to local imbalances in the model fields some fields will drift away from the analysis series with time only to be reset at the next data insertion cycle.
The analysis system itself can also be subject to 'data shock' when particular data types are only available at certain times and their intermittent presence establishes dynamic, hydrologic and thermodynamic balances that are not consistent with those of the model. This behaviour was exacerbated by the fact that the $\mathrm{Bu}$ reau's operational assimilation scheme during EOP3 was a statistical interpolation scheme in which water vapour was independent of the dynamical variables. The binning technique can be anticipated to be sensitive to systematic model spin-up which will introduce 'jumps' in the time series at the times the forecast series are concatenated to form the EOP3 period. For example, since the ASSIM forecasts are explicitly 'tied' to the analysis series every 6 hours a saw tooth pattern can be induced by systematic model error adding harmonics which could confuse the interpretation of the diurnal behaviour. This behaviour is most pronounced for humidity variables in data sparse regions.

\section{Comparing the surface pressure time series}

\subsection{Basic statistics}

Figure 2 shows the scatter plots of the model time series for EOP3 against the in-situ data and Table 2 shows the standard error information. Note that no corrections have been made for the difference in altitude between the model and the measurement sites as shown in Table 1. In most cases the bias accounts for most of the RMS difference and this in turn is almost certainly mostly due to the elevation differences. There is a noticeable spread in the forecast relative to the assimilation series at Lindenberg as evidenced by the large increase in variance and in the RMS difference with the in-situ data. Overall the Lindenberg site and the SGP locations have the best correlation with the in-situ data, with the smallest correlations occurring for the data sparse sites, Equatorial Island and WPO. The large difference in elevation for Equatorial Island results in a very large bias and corresponding RMS difference relative to the CEOP reference site. Using the geopotential from the model to estimate the pressure at the elevation of the station still results in a bias of about $20 \mathrm{hPa}$ and no substantive reduction in the correlation. Overall, the variance in the model is larger than that of the 


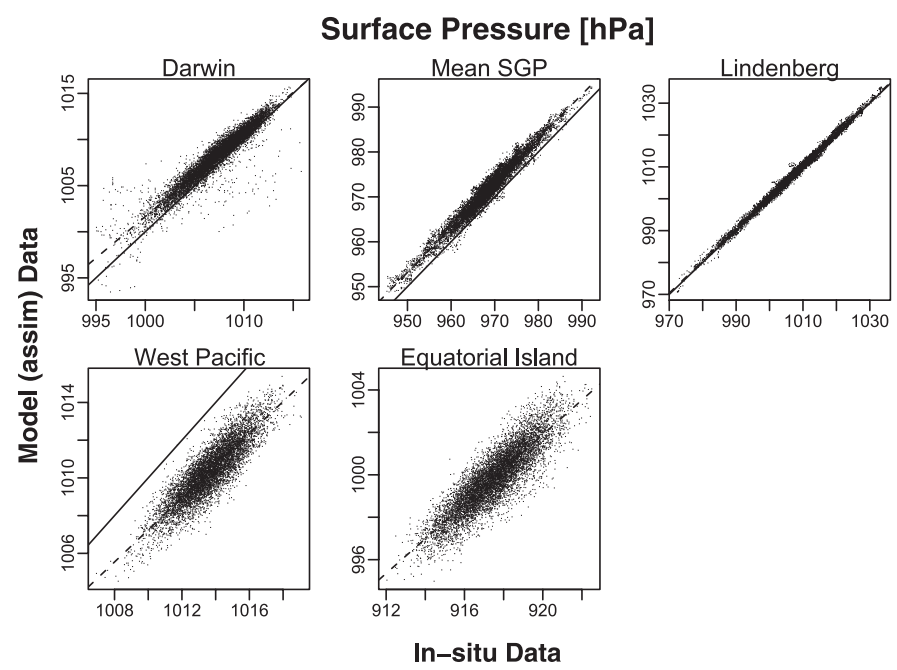

Fig. 2. Scatter plots of model surface pressure against the in-situ data for the ASSIM model time series for each MOLTS site. The solid line is the 45 degree line through the origin and the dashed line is a linear regression fit to the data. There are no pressure data for the MDB.

Table 2. Comparative statistics for surface pressure in $\mathrm{hPa}$ for the hourly forecast series during the assimilation cycle and from the 12 to 36 hour forecasts against the in-situ data for the selected sites where data was available. The individual series variance is given in the last three columns. Note that the variances for the model for Ringwood differ from those given for ARM-SGP because missing data times at Ringwood are excluded from the model as well. The original unfiltered Darwin statistics are given in the last row.

\begin{tabular}{|l|c|c|c|c|c|c|c|c|c|}
\hline & \multicolumn{3}{|c|}{ Assimilation } & \multicolumn{3}{c|}{ Forecast } & \multicolumn{3}{c|}{ Variance } \\
\hline & RMS & BIAS & Corr & RMS & BIAS & Corr & In-situ & Assim & Forc \\
\hline Lindenberg & 0.887 & 0.374 & 0.996 & 1.900 & -0.225 & 0.984 & 85.26 & 85.67 & 100.81 \\
\hline ARM-SGP & 3.292 & 3.000 & 0.981 & 3.585 & 2.529 & 0.934 & 46.66 & 47.69 & 49.93 \\
\hline Equatorial Island & 82.350 & 82.346 & 0.872 & 82.448 & 82.444 & 0.862 & 2.51 & 2.21 & 2.39 \\
\hline WPO & 3.391 & -3.299 & 0.874 & 3.275 & -3.160 & 0.847 & 2.49 & 2.36 & 2.34 \\
\hline ARM-Darwin & 1.359 & 0.912 & 0.943 & 1.489 & 0.855 & 0.916 & 9.14 & 8.29 & 8.47 \\
\hline Ringwood (SGP) & 4.528 & 4.386 & 0.988 & 4.590 & 3.983 & 0.950 & 51.43 & 49.44 & 52.06 \\
\hline ARM-Darwin (Orig) & 1.590 & 0.946 & 0.915 & 1.687 & 0.889 & 0.893 & 10.08 & 8.29 & 8.47 \\
\hline
\end{tabular}

in-situ data for the mid-latitude locations but smaller in the tropics.

The original Darwin in-situ data had a number of extreme low and high pressures during the November to February monsoon period. Often these extreme pressure values are associated with precipitation and the values and the magnitude of the changes over such short times indicates that they are probably attributable to known problems with the sensor as docu- mented in the site meta data available on the JOSS web site. These points were replaced by values obtained by linearly interpolation of the surrounding rain-free times. Table 2 has an extra row to show the statistics of the raw hourly data.

Figure 3 shows the comparison between Ringwood and the mean SGP data. The scatter plot is slightly more compact than the ASSIM against mean SGP plot in Fig. 2. The correla- 


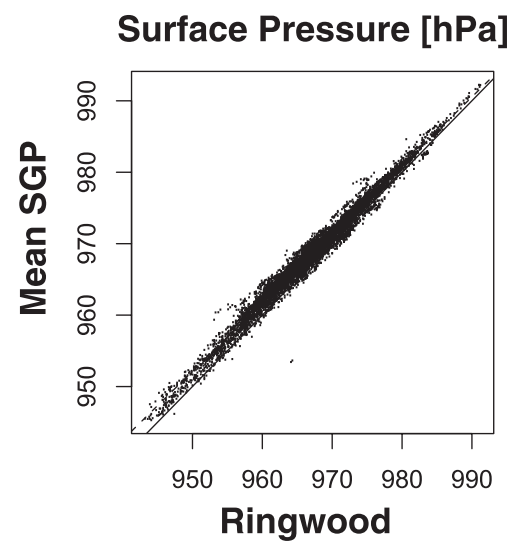

Fig. 3. Scatter plot for the in-situ surface pressure from the mean SGP site against the data from the Ringwood station. tion of 0.987 is comparable to that for ASSIM (0.981) and the variability is close to the model's suggesting that for surface pressure the differences between model and observations are of about the same order as the variability over the grid scale as represented by the different stations, at least in the absence of convection.

\subsection{Global wavelet power spectra}

Figure 4 shows the global wavelet power spectra for surface pressure. The structures are very similar between the in-situ data and the model with the exception of Equatorial Island where the model has an extra peak at a period of about 55 days. There appears to be a tendency for the FORC series to have more strength for larger periods with possibly less strength in the 20 to 80 day periods. Figure 5 shows the short period part of the spectra for the tropical locations; while Lindenberg and

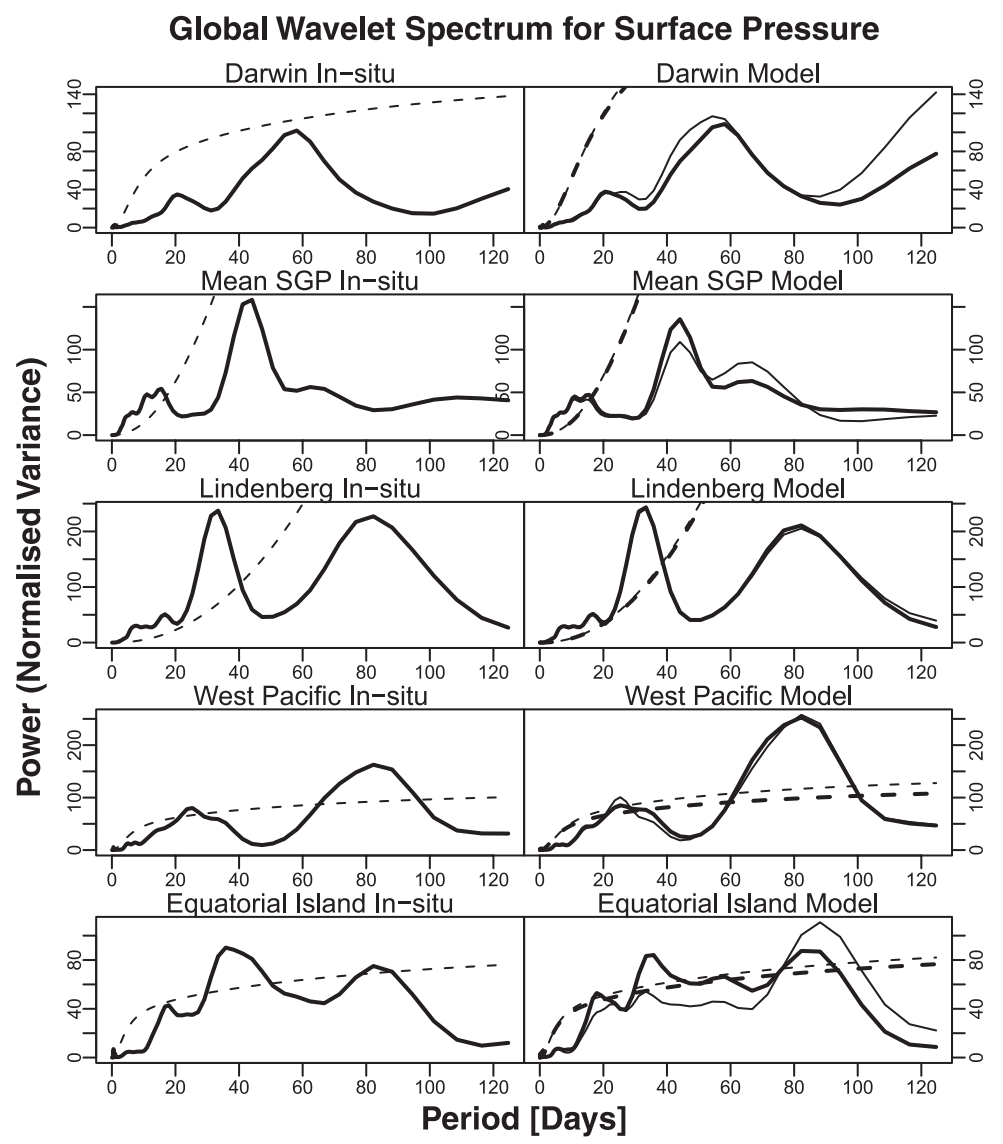

Fig. 4. The global wavelet power spectra for surface pressure for the in-situ data (left column) and the model (right). The ASSIM series is the heavy line on the right and the thin line the FORC series. The dashed lines are the corresponding line of statistical significance at the $5 \%$ level. 


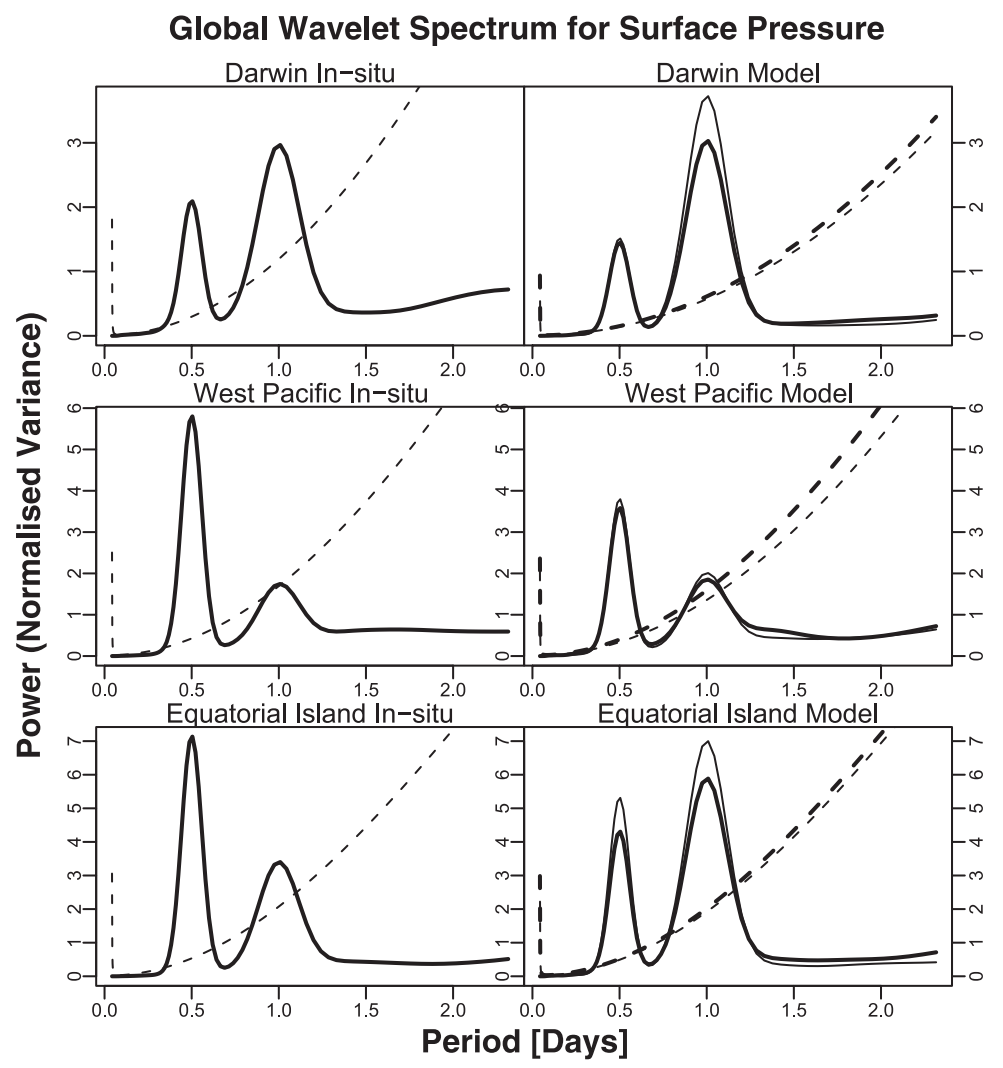

Fig. 5. The short period section of the global wavelet power spectra for surface pressure shown in figure 4 .

the SGP show significant power over the 1 to 20 day range neither has any real power in the diurnal cycle. Note that the diurnal mode dominates the semi-diurnal mode at Darwin but that the reverse is true at WPO and Equatorial Island. Both model time series reproduce that behaviour for Darwin and WPO. The model FORC series has a stronger diurnal signal relative to ASSIM for Darwin and Equatorial Island, possibly due to its construction from concatenated 24 hour segments.

\subsection{Annual average diurnal variation}

Figure 6 shows the annual mean diurnal variation of surface pressure for the model and the observations illustrating the expected combination of diurnal and semi-diurnal signals (Dai and Wang 1999) with the strongest amplitude at the tropical sites and the weakest signal at Lindenberg. At all locations the major morning peak is underestimated while the minor evening peak is well simulated. Due to the weakness of the signal at Lindenberg the model ASSIM series shows sensitivity to the data insertions at $0,6,12$ and 18 UTC where the individual 6 hour forecasts are joined. These are not present in the FORC series because the 12 to 36 hour forecasts are continuous during the day with the individual forecasts joined at 0 UTC. It should be noted that one reason the diurnal signal is as strong in the model data as in the in-situ data is due to the use of incremental normal mode initialisation which preserves this mode while removing gravity waves (Seaman et al. 1995).

\subsection{Summary}

The results of the previous sections show that surface pressure appears to be very similar in the model and the in-situ data and thus seems to be essentially independent of the spatial resolution and the physical differences between the model and real world sites. All observed frequency modes appear to be well rep- 


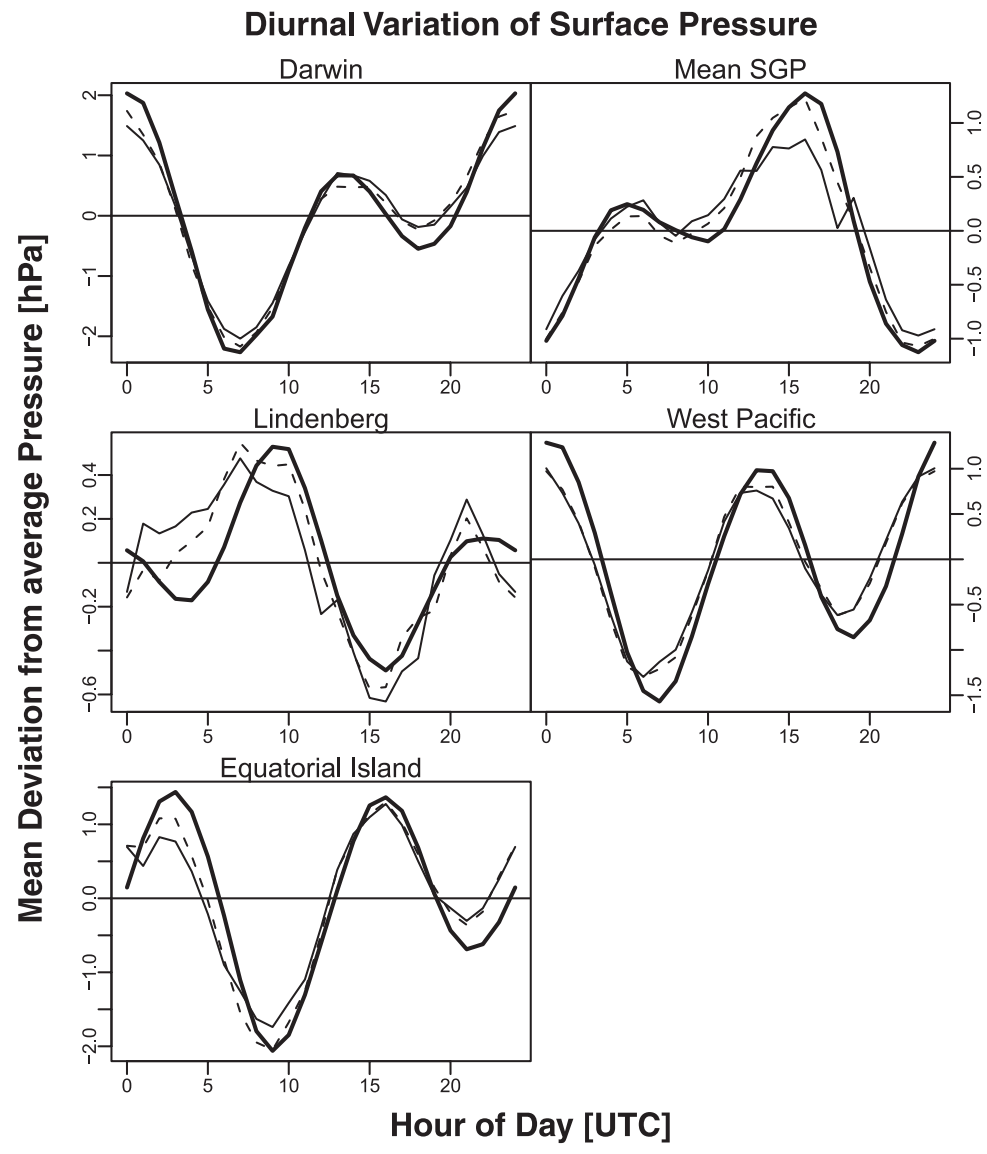

Fig. 6. The diurnal variation of surface pressure for the MOLTS sites. The thick solid curves are the in-situ data, the thin lines the ASSIM and the dashed line the FORC results from the binning method described in the text.

resented, although the scatter plots show some spread and the presence of 'anomalous' extremes. In the next section the methodology will be applied to model variables which show some resolution dependence and sensitivity to the physical properties of the location.

\section{Other variables}

\subsection{Screen temperature}

Screen temperature is determined in the model as an extrapolation from the lowest model level and is sensitive to the surface properties such as those that determine the surface temperature (at least over land; the model's SST data does not vary diurnally). Figure 7 shows the scatter plots for screen temperature and Table 3 shows the corresponding statistics. As the relative correlations indicate the ASSIM and FORC series are very close in all of the fol- lowing comparisons. As noted above there is a discrepancy between the model screen height and most of the in-situ sites. In fact, even though the actual sensor height is $2 \mathrm{~m}$ at the Lindenberg and the SGP and MDB sites the model shows quite good agreement with the insitu data with only a slightly larger variance in the forecast. Figure 8 shows the two scatter plots of the mean SGP and MDB with the appropriate single station data. The comparison of these results with Fig. 7 suggest that the model-observation differences are larger than the variability across the different stations within the dataset, which is confirmed by the correlation coefficients of 0.994 and 0.987 respectively for Ringwood and Kyeamba against the mean site data as compared with 0.962 and 0.939 for the model. This is to be expected as surface temperature is determined by the 


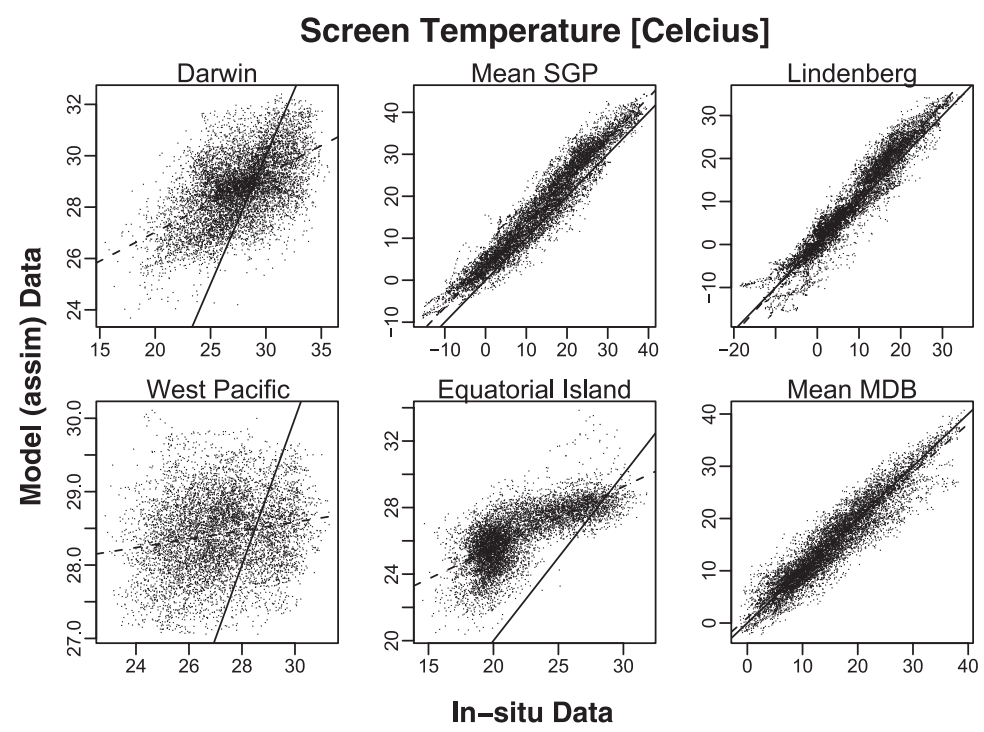

Fig. 7. Scatter plots of model screen temperature (nominally at $1.5 \mathrm{~m}$ ) against the in-situ data for the ASSIM time series for each location. The solid line is the 45 degree line through $(0,0)$ and the dashed line is a simple linear regression. The plots for the FORC series are very similar to those shown for the ASSIM time series and are not shown.

Table 3. Comparative statistics for the $1.5 \mathrm{~m}$ level temperature in $\mathrm{K}$ for the hourly forecast series during the assimilation cycle and from the 12 to 36 hour forecasts against the in-situ data for the selected sites where data was available. The individual series variance is given in the last three columns.

\begin{tabular}{|l|c|c|c|c|c|c|c|c|c|}
\hline & \multicolumn{3}{|c|}{ Assimilation } & \multicolumn{3}{c|}{ Forecast } & \multicolumn{3}{c|}{ Variance } \\
\hline & RMS & BIAS & In-situ & RMS & BIAS & Corr & In-situ & Assim & Forc \\
\hline Lindenberg & 3.279 & 1.239 & 0.962 & 3.912 & 2.189 & 0.959 & 92.93 & 115.81 & 121.24 \\
\hline ARM-SGP & 4.748 & 3.496 & 0.962 & 5.742 & 4.327 & 0.951 & 126.50 & 137.17 & 147.31 \\
\hline Equatorial Island & 4.908 & 4.300 & 0.717 & 4.874 & 4.255 & 0.719 & 10.64 & 2.83 & 2.66 \\
\hline MDB & 2.777 & -0.125 & 0.939 & 3.025 & 0.134 & 0.928 & 64.03 & 62.60 & 63.00 \\
\hline WPO & 2.047 & 1.237 & 0.182 & 2.009 & 1.166 & 0.188 & 2.70 & 0.28 & 0.34 \\
\hline ARM-Darwin & 2.923 & 1.088 & 0.547 & 2.986 & 1.067 & 0.496 & 10.24 & 1.71 & 1.86 \\
\hline Ringwood (SGP) & 4.277 & 2.560 & 0.959 & 5.231 & 3.378 & 0.948 & 139.24 & 146.53 & 157.82 \\
\hline Kyeamba (MDB) & 3.175 & -1.140 & 0.935 & 3.321 & -0.880 & 0.924 & 69.92 & 62.60 & 63.00 \\
\hline
\end{tabular}

surface energy balance which in turn depends on the surface properties. The model's globally constant grid average climatological values are not optimal for the sites examined here emphasizing the need for a more sophisticated land surface parameterization.

For Darwin the model systematically underestimates high temperatures and overesti- mates low temperatures relative to the in-situ data. For model grid points near the ocean the assumption of constant sea surface temperature (SST) over the entire day will act to suppress the expected diurnal variation at screen height because the surface temperature is the main source of heat for the PBL. This results in the reduced amplitude of the diurnal signal 
Screen Temperature [Celcius]
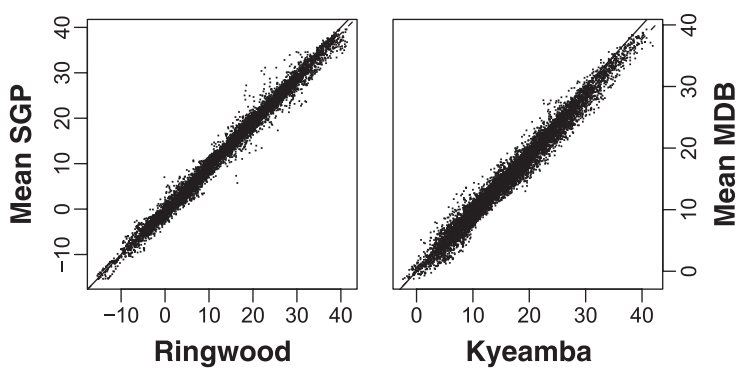

Fig. 8. Scatter plot for the in-situ screen temperature from the mean SGP site against the data from the Ringwood station (left) and the mean MDB site data against that from the Kyeamba station. of screen level temperature shown in the annual diurnal variation shown in Fig. 9 for the three locations close to ocean points. The WPO site shows little correlation between the model and in-situ data and this is not surprising; the model point is a sea point with a constant SST as the lower boundary resulting in an essentially flat diurnal signal, while the weather at the real observational site should be heavily influenced by the presence of Peleliu Island. The scatter plot for Equatorial Island shows a curious pattern where the model systematically overestimates low temperatures up to about $28^{\circ} \mathrm{C}$ but then appears to strike a threshold where it flattens out. This was not the case for night time when the model temperatures are essentially uncorrelated with the in-situ data. The overall shape of the scatter plot and the correlation do not depend on model wind direc-

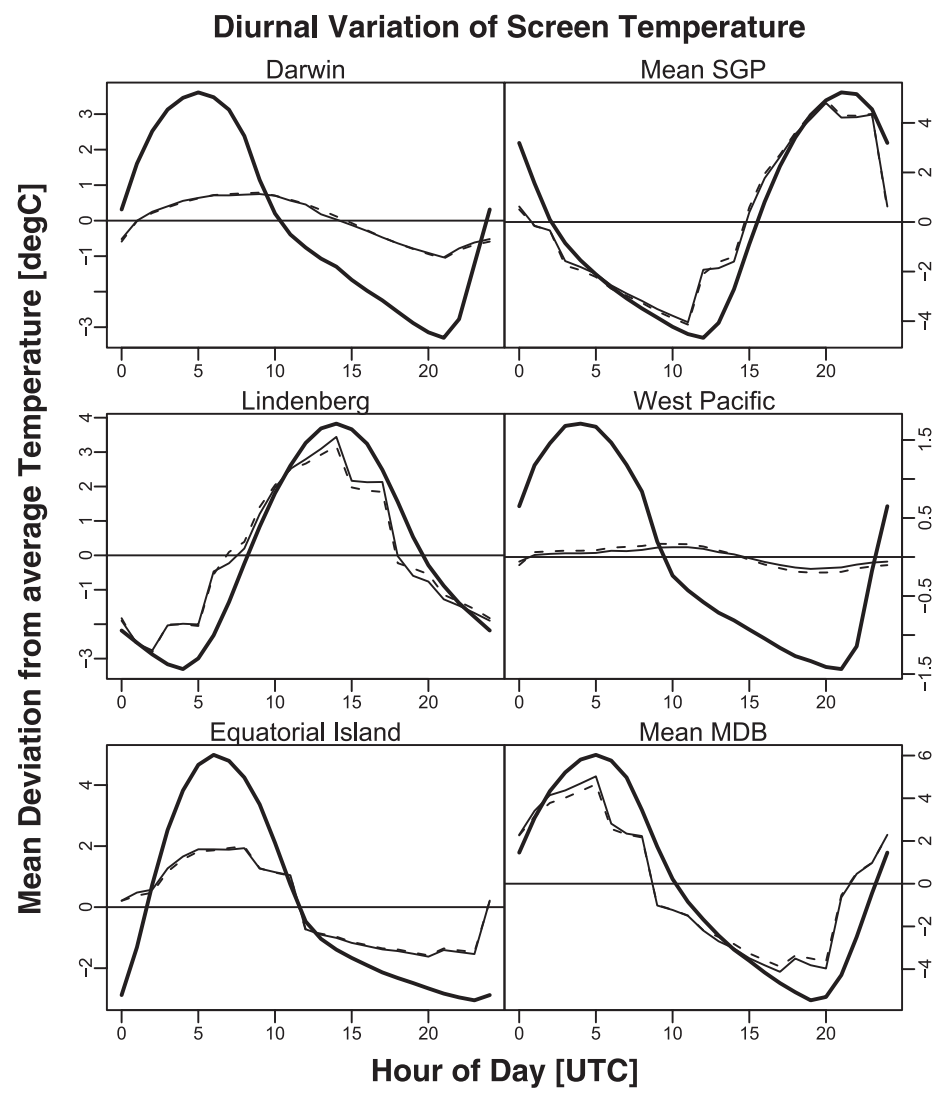

Fig. 9. The diurnal variation of screen temperature for the MOLTS sites. The thick solid curves are the in-situ data, the thin lines the ASSIM and the dashed line the FORC results from the binning method described in the text. 
Global Wavelet Spectrum for Screen Temperature

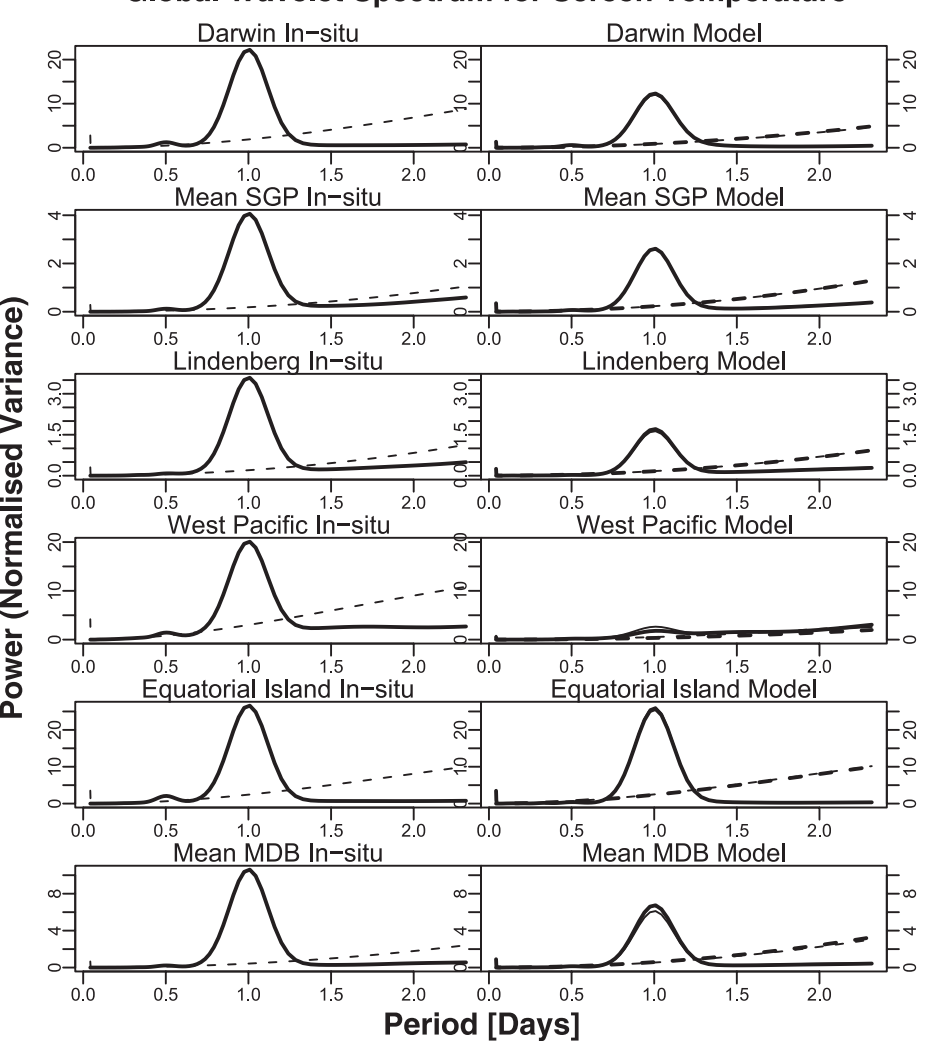

Fig. 10. The short period global wavelet power spectrum for screen temperature for the in-situ data (left column) and the model (right). The ASSIM series is the heavy line on the right and the thin line the FORC series. The dashed lines are the corresponding line of statistical significance at the $5 \%$ level.

tion, season or daylight hour and warrant further investigation. The model bias of $4.3^{\circ} \mathrm{C}$ is consistent with the difference between the model's surface temperature and that at the station elevation which is $3.9^{\circ} \mathrm{C}$ on average.

Even in the data rich regions of the SGP and Lindenberg the model signal shows discontinuities every 3 hours or so which can be attributed to the radiative transfer scheme which keeps the net radiation fields constant between calculations 3 hours apart. The flatness of the model signal at Darwin suggests the model is dominated by the presence of constant SSTs to a much larger extent than the observations.

Figure 10 shows the short period section of the global wavelet spectra for screen height temperature. All locations have consistent diurnal peaks consistent with the domination of solar radiation in the surface energy budget. There are substantial differences in the amount of variance explained by the model and the observations; except for Equatorial Island the model underestimates the diurnal strength in some cases substantially. This is indicative of the differences in the distribution of variance over longer periods which are not shown here due to the lack of statistically significant peaks.

\subsection{Screen level moisture}

Table 4 shows the statistics for specific humidity at the model height of $1.5 \mathrm{~m}$ (the sensor heights are the same as for temperature) and Fig. 11 shows the scatter plots for the different locations. The specific humidity modelobservation differences are mostly larger than for temperature with essentially no agreement between model and observations for Equatorial Island as would be expected from the difference in physical character between the model and 
Table 4. Comparative statistics for the $1.5 \mathrm{~m}$ level specific humidity in $\mathrm{g} \mathrm{kg}^{-1}$ for the hourly forecast series during the assimilation cycle and from the 12 to 36 hour forecasts against the in-situ data for the selected sites where data was available. The individual series variance is given in the last three columns.

\begin{tabular}{|l|c|c|c|c|c|c|c|c|c|}
\hline & \multicolumn{3}{|c|}{ Assimilation } & \multicolumn{3}{c|}{ Forecast } & \multicolumn{3}{c|}{ Variance } \\
\hline & RMS & BIAS & In-situ & RMS & BIAS & Corr & In-situ & Assim & Forc \\
\hline Lindenberg & 1.45 & -0.072 & 0.844 & 1.81 & -0.078 & 0.739 & 7.21 & 4.54 & 4.08 \\
\hline ARM-SGP & 2.79 & -0.912 & 0.832 & 3.1 & -0.726 & 0.756 & 21.15 & 9.95 & 10.89 \\
\hline Equatorial Island & 4.16 & 3.824 & 0.366 & 4.05 & 3.729 & 0.377 & 2.39 & 1.79 & 1.60 \\
\hline MDB & 1.87 & 0.438 & 0.640 & 1.83 & 0.530 & 0.647 & 3.23 & 5.47 & 5.10 \\
\hline WPO & 1.75 & 1.429 & 0.342 & 1.68 & 1.284 & 0.313 & 0.62 & 0.90 & 1.06 \\
\hline ARM-Darwin & 3.84 & 2.586 & 0.691 & 3.77 & 2.468 & 0.695 & 13.41 & 12.03 & 13.37 \\
\hline Kyeamba (MDB) & 2.214 & 0.558 & 0.527 & 2.170 & 0.651 & 0.539 & 0.00 & 5.47 & 5.10 \\
\hline
\end{tabular}

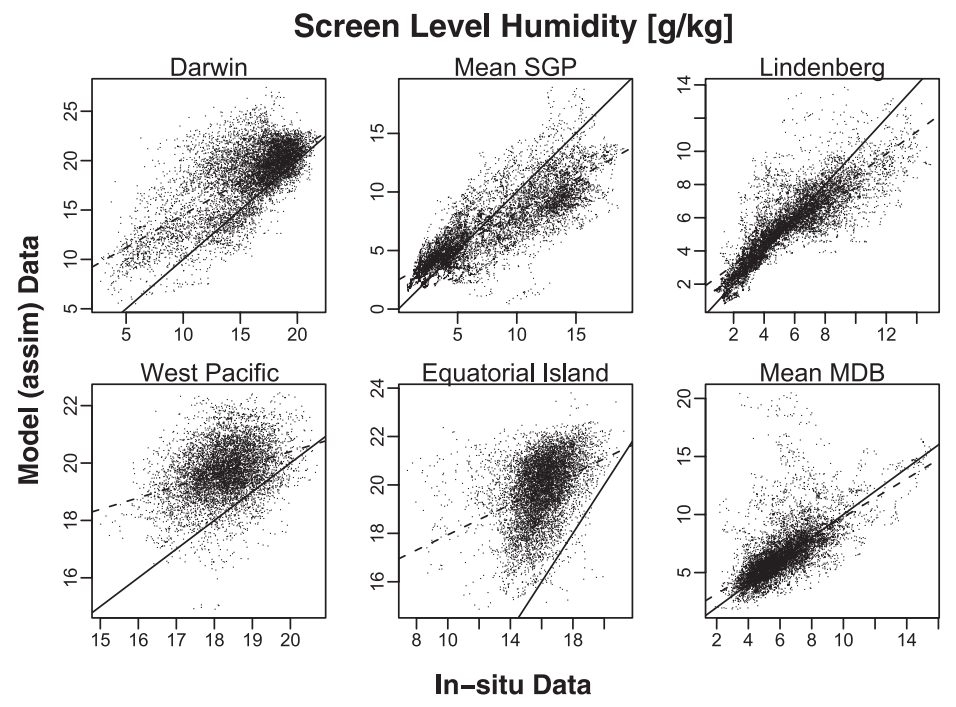

Fig. 11. Scatter plots of model screen level specific humidity (nominally at $1.5 \mathrm{~m}$ ) against the in-situ data for the ASSIM time series for each site. The solid line is the 45 degree line through $(0,0)$ and the dashed line is a linear regression. The plots for the FORC series are very similar to those shown for the ASSIM time series and are not shown.

the observation site. The scatter plot for WPO shows a distinct wet bias but the model and in-situ data are much more similar than was the case for screen temperature. This is also true for Darwin. The agreement with the mean MDB site is also much worse than might be expected from the comparison of temperature because of a distinct 'tongue' of too moist points relative to the observations. This behavior of 'anomalous' outliers is also a feature of the mean SGP and Lindenberg locations. For the mid-latitude sites the model tends to have much smaller variance than the in-situ data although not for Kyeamba perhaps reflecting the greater variability of a single site.

Figure 12 shows the diurnal part of the global wavelet spectrum for each location. Darwin, Equatorial Island, WPO and the MDB locations show pronounced and statistically significant diurnal peaks with some differences 
Global Wavelet Spectrum for Screen Level Humidity
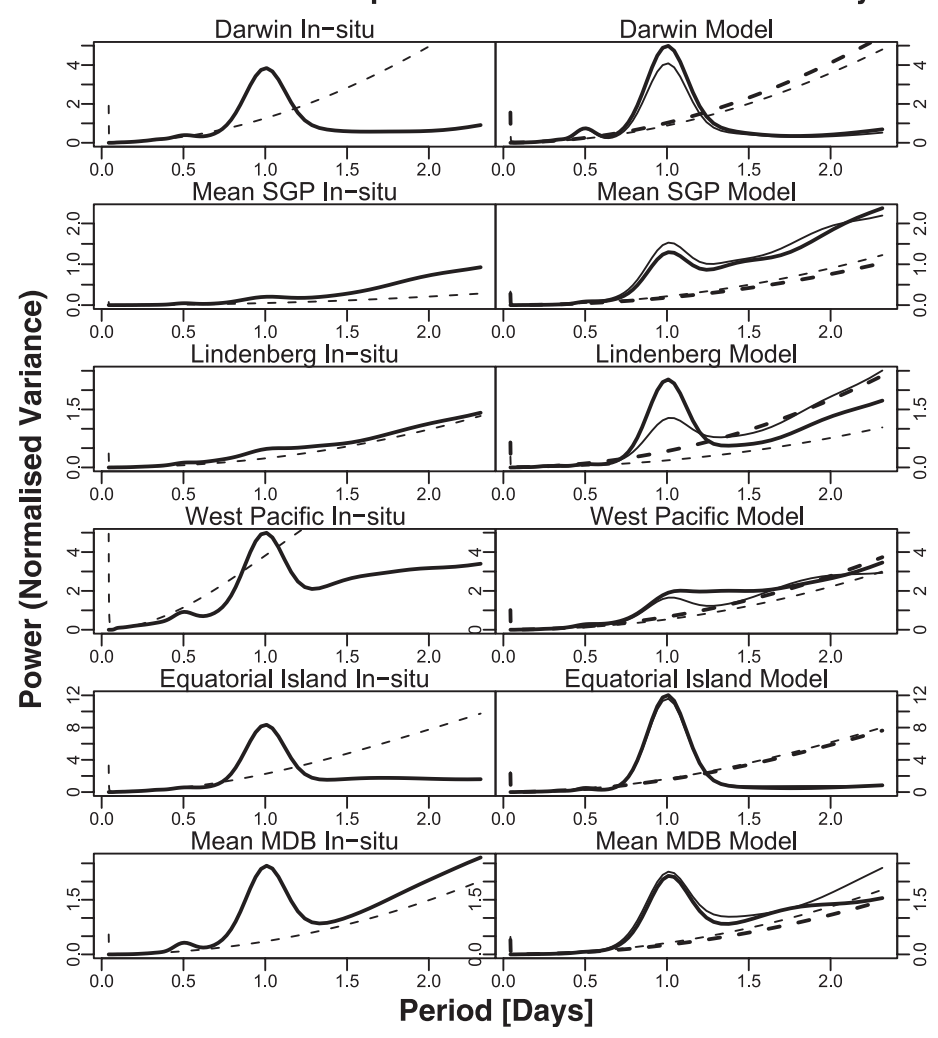

Fig. 12. The global wavelet power spectrum for screen level specific humidity for the in-situ data (left column) and the model (right). The ASSIM series is the heavy line on the right and the thin line the FORC series. The dashed lines are the corresponding line of statistical significance at the $5 \%$ level.

in the variance between the model and in-situ data. For Lindenberg and the mean SGP the model series both have a strong diurnal peak which is almost completely absent from the observations. WPO is the only location to show statistically significant peaks of variability of period longer than 20 days with the model giving the longer periods much more relative strength than the observations and shifting the power to lower frequencies.

Figure 13 shows the annual mean diurnal variation for the screen level specific humidity. Paradoxically the best model result is for Equatorial Island where the model and in-situ physical locations are very different as discussed in Section 2.1. The variation at the SGP is almost in phase but lacks the semi-diurnal component which is also missing at Lindenberg and the mean MDB location. The signal at Darwin is out of phase by about 9 hours which is an indication that the convection which dominates the moisture field there is triggering too early in the day in the model physics. This is a well known problem (Yang and Slingo 2001) with models using parameterized convection.

\subsection{Wind speed}

The statistics for $10 \mathrm{~m}$ wind speed given in Table 5 and the scatter plots in Fig. 14 show that the scatter between model and observations is almost random for Darwin but quite reasonable for the mean SGP and MDB and Lindenberg locations. The correlation between the model and observations is generally lower than for screen level humidity except for the mean MDB and Kyeamba locations. The model tends to have greater variance than the in-situ data except for the single site of Ringwood. 


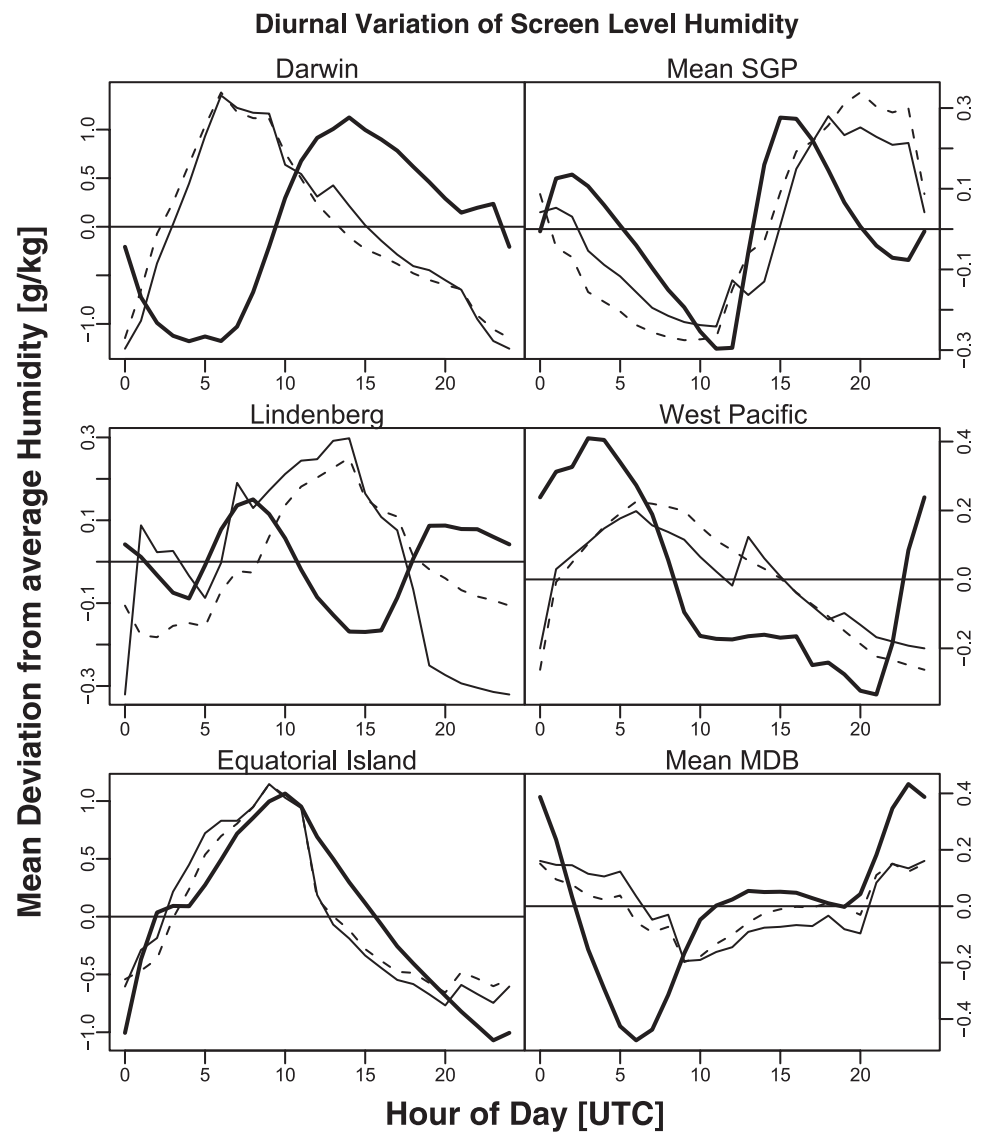

Fig. 13. The diurnal variation of screen level specific moisture for the MOLTS sites. The thick solid curves are the in-situ data, the thin lines the ASSIM and the dashed line the FORC results from the binning method described in the text.

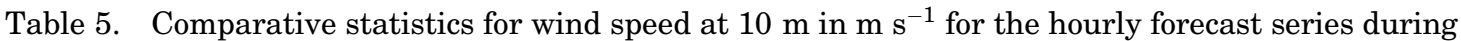
the assimilation cycle and from the 12 to 36 hour forecasts against the in-situ data for the selected sites where data was available. The individual series variance is given in the last three columns.

\begin{tabular}{|l|c|c|c|c|c|c|c|c|c|}
\hline & \multicolumn{3}{|c|}{ Assimilation } & \multicolumn{3}{c|}{ Forecast } & \multicolumn{3}{c|}{ Variance } \\
\hline & RMS & BIAS & Corr & RMS & BIAS & Corr & In-situ & Assim & Forc \\
\hline Lindenberg & 1.4 & -0.077 & 0.763 & 1.53 & 0.078 & 0.714 & 3.60 & 4.44 & 4.46 \\
\hline ARM-SGP & 1.51 & -0.436 & 0.751 & 1.8 & -0.247 & 0.627 & 3.51 & 4.66 & 4.84 \\
\hline MDB & 1.5 & 0.454 & 0.789 & 1.61 & 0.530 & 0.738 & 1.80 & 4.97 & 4.88 \\
\hline ARM-Darwin & 3.39 & 1.760 & 0.105 & 3.8 & 2.207 & 0.051 & 2.61 & 6.66 & 7.41 \\
\hline Ringwood (SGP) & 1.994 & -0.612 & 0.668 & 2.249 & -0.443 & 0.553 & 5.93 & 4.74 & 4.87 \\
\hline Kyeamba (MDB) & 1.846 & 0.557 & 0.626 & 1.946 & 0.633 & 0.579 & 2.77 & 4.97 & 4.88 \\
\hline
\end{tabular}




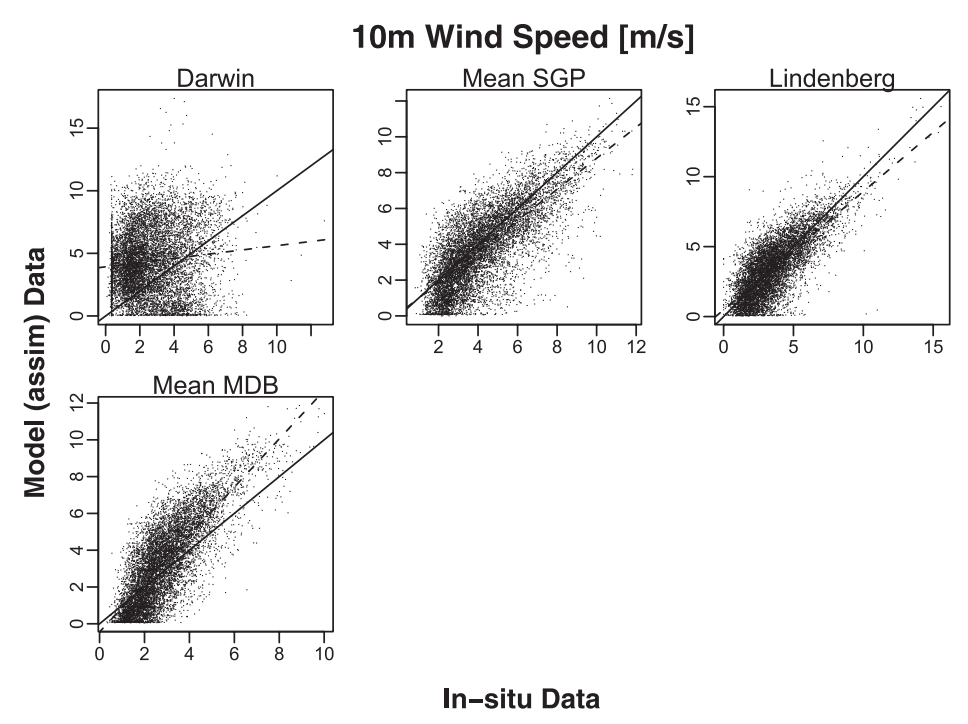

Fig. 14. Scatter plots of model surface wind speed (nominally at $10 \mathrm{~m}$ ) against the in-situ data for the ASSIM time series for each site. The solid line is the 45 degree line through $(0,0)$ and the dashed line is a linear regression.

Whether this is due to the fact that the in-situ wind fields are hourly means and the model fields are instantaneous or whether the model data is inherently more noisy requires more investigation. Figure 15 shows the global power spectra for the four locations which have $10 \mathrm{~m}$ wind data. All have a significant diurnal signal with the model peaks being generally lower in power than the in-situ data. Figure 16 shows the only spectra with significant peaks at longer periods. In the Darwin data, both the model and station data show a significant peak in power broadly centered on the 45 day period although its amplitude in the model is about an order of magnitude stronger. The model also overestimates the strength of the peak at around a period of 20 days although in this case the model peak is not statistically significant. The longer period regimes are completely different. The Lindenberg observations and the model exhibit peaks at about the same periods although their relative strengths are substantially different and have less statistical significance for the model time series. The model has an extra peak at about 20 days but misses the peak at about 65 days, particularly the FORC series.

Figure 17 shows the corresponding mean annual diurnal wind speed variations. The annual mean diurnal cycle at Darwin is completely out of phase with the observations. The mean SGP, MDB and Lindenberg are in quite good agreement with the observational data but also show discontinuities in both the model ASSIM and FORC series which suggests that they are not due to data insertion effects. The discontinuities are too numerous to correspond to the radiation time step effect noted in the screen temperatures and undoubtedly contribute to the larger model variances.

Overall the Darwin site has by far the lowest correlations and the greatest differences from the model data. This could be a consequence of the fact that it is the location with the largest differences in the diurnal signal; the amplitude of the diurnal signal is $1 \mathrm{~m} \mathrm{~s}^{-1}$ and this combined with the completely out-of-phase character should lead to overall differences of more than $2 \mathrm{~m} \mathrm{~s}^{-1}$ at certain times of the day. The two mean sites have RMS differences of around $1.5 \mathrm{~m} \mathrm{~s}^{-1}$ and biases of less than $0.5 \mathrm{~m} \mathrm{~s}^{-1}$ in magnitude which is quite reasonable considering the differences between the real vegetation's surface roughness and the model's climatological constant values. The comparison of the mean SGP site against the single station data for Ringwood has quite similar spread to the ASSIM model results although the correlation coefficient is larger (0.827 versus 0.751$)$. In contrast the scatter of mean MDB data 


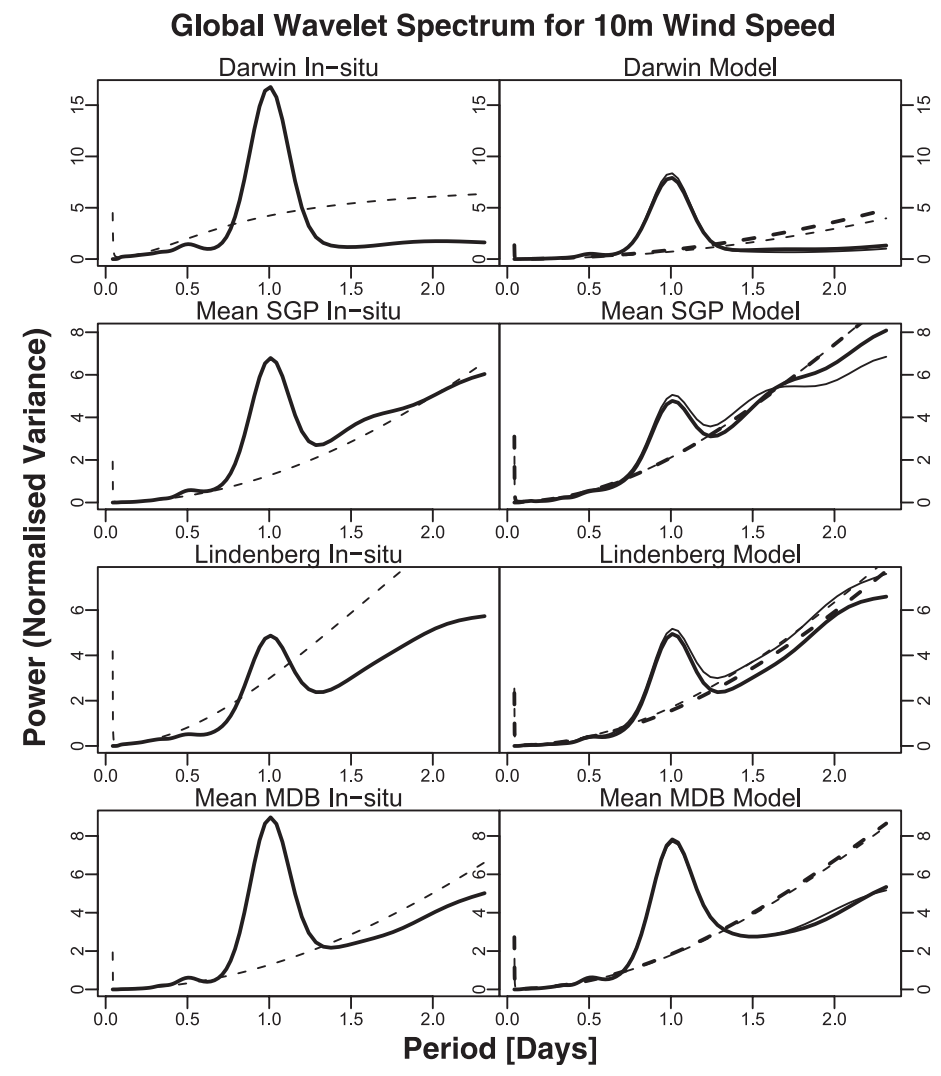

Fig. 15. The short period section of the global wavelet power spectrum for $10 \mathrm{~m}$ wind speed for the in-situ data (left column) and the model (right). The ASSIM series is the heavy line on the right and the thin line the FORC series. The dashed lines are the corresponding line of statistical significance at the $5 \%$ level.

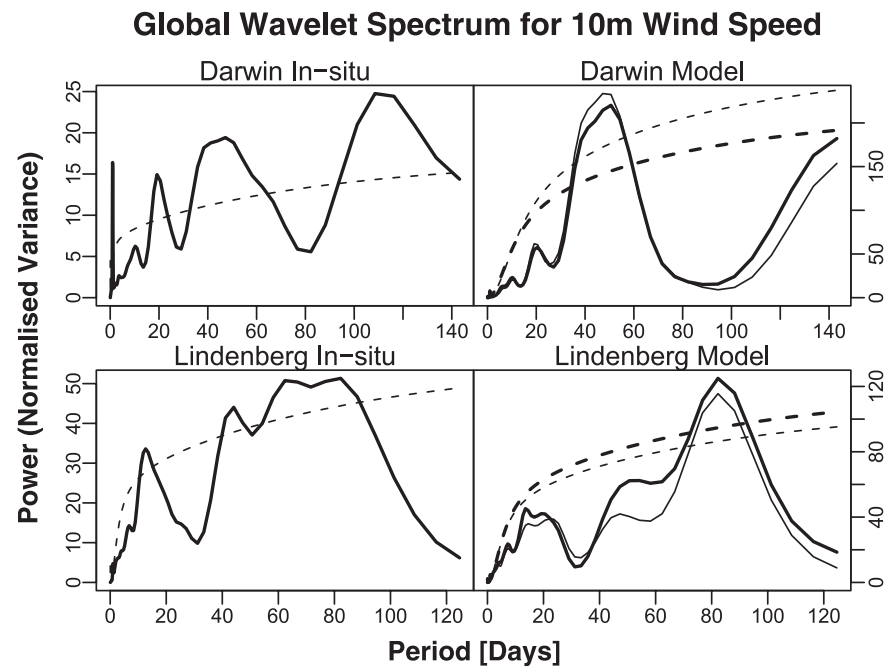

Fig. 16. The global wavelet power spectrum for $10 \mathrm{~m}$ wind speed for the in-situ data (left column) and the model (right) showing the longer periods. The ASSIM series is the heavy line on the right and the thin line the FORC series. The dashed lines are the corresponding line of statistical significance at the $5 \%$ level. Note the difference in the $y$-axis scales between in-situ and model plot. 


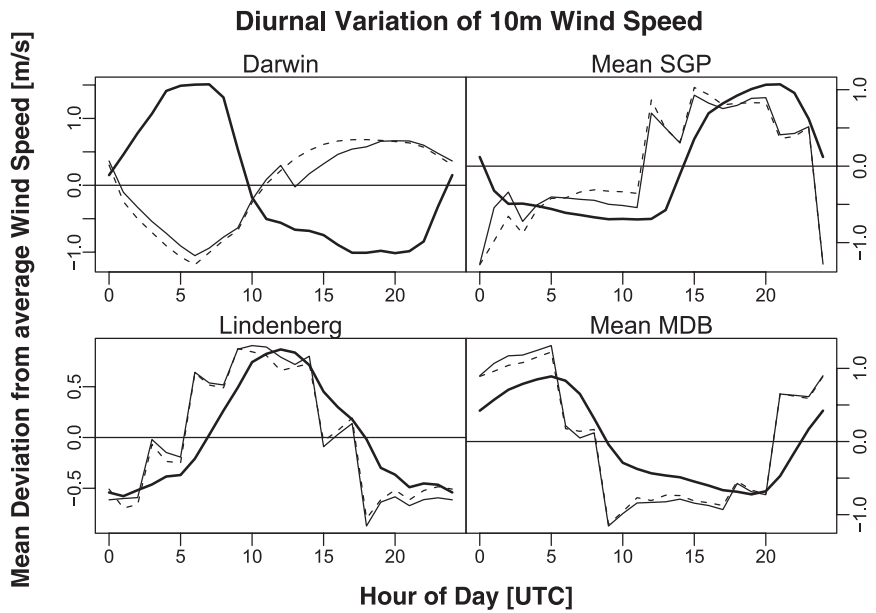

Fig. 17. The annual mean diurnal variation of $10 \mathrm{~m}$ wind speed for the MOLTS sites. The thick solid curves are the in-situ data, the thin lines the ASSIM and the dashed line the FORC results from the binning method described in the text.

against Kyeamba is actually worse (0.705 versus 0.789).

\subsection{Precipitation}

The local nature of precipitation and its intermittent temporal nature makes a direct comparison of the hourly time series problematic at best. Instead the analysis will concentrate on the monthly means and the annual total as well as the wavelet power spectra. The model precipitation is an accumulation over the previous model forecast hour. Hence the ASSIM precipitation is written out during the six hour forecasts connecting the analysis cycles and should be more sensitive to the models hy- drological cycle spin-up/down than that of the forecast series. When in-situ data was flagged as missing the corresponding model data was ignored in the statistical analysis but for the wavelet analyses both were set to zero to minimise missing data edge effects. Some simple statistics for a comparison of the monthly means and the ratio of model to in-situ EOP3 totals are presented in Table 6 . The model overestimates the annual rainfall in all cases except for ASSIM for the mean SGP and for Darwin which is underestimated by more than two thirds. It does, however, reproduce the concentration of the Darwin rainfall over the southern summer months and this is responsible for the

Table 6. Error statistics for precipitation for the assimilation (ASSIM), 12-36 hour forecasts (FORC) and (where present) the station nearest to the model's MOLTS point. The Ann. Ratio is the ratio of the annual rainfall to the annual rainfall at the site. The correlations are the monthly correlations with the site rainfall.

\begin{tabular}{|l|c|c|c|c|c|c|}
\hline & \multicolumn{2}{|c|}{ ASSIM } & \multicolumn{2}{c|}{ FORC } & \multicolumn{2}{c|}{ STATION } \\
\hline & Ann. Ratio & Correlation & Ann. Ratio & Correlation & Ann. Ratio & Correlation \\
\hline Lindenberg & 1.011 & 0.513 & 1.327 & 0.579 & & 0.902 \\
\hline ARM-SGP & 0.726 & 0.283 & 1.043 & 0.589 & 0.864 & \\
\hline Equatorial Island & 1.534 & 0.442 & 1.284 & 0.401 & & 0.847 \\
\hline MDB & 1.183 & 0.837 & 1.865 & 0.730 & 1.172 & \\
\hline WPO & 1.582 & 0.750 & 1.571 & 0.725 & & \\
\hline ARM-Darwin & 0.296 & 0.821 & 0.243 & 0.900 & & \\
\hline
\end{tabular}




\section{Global Wavelet Spectrum for Precipitation}
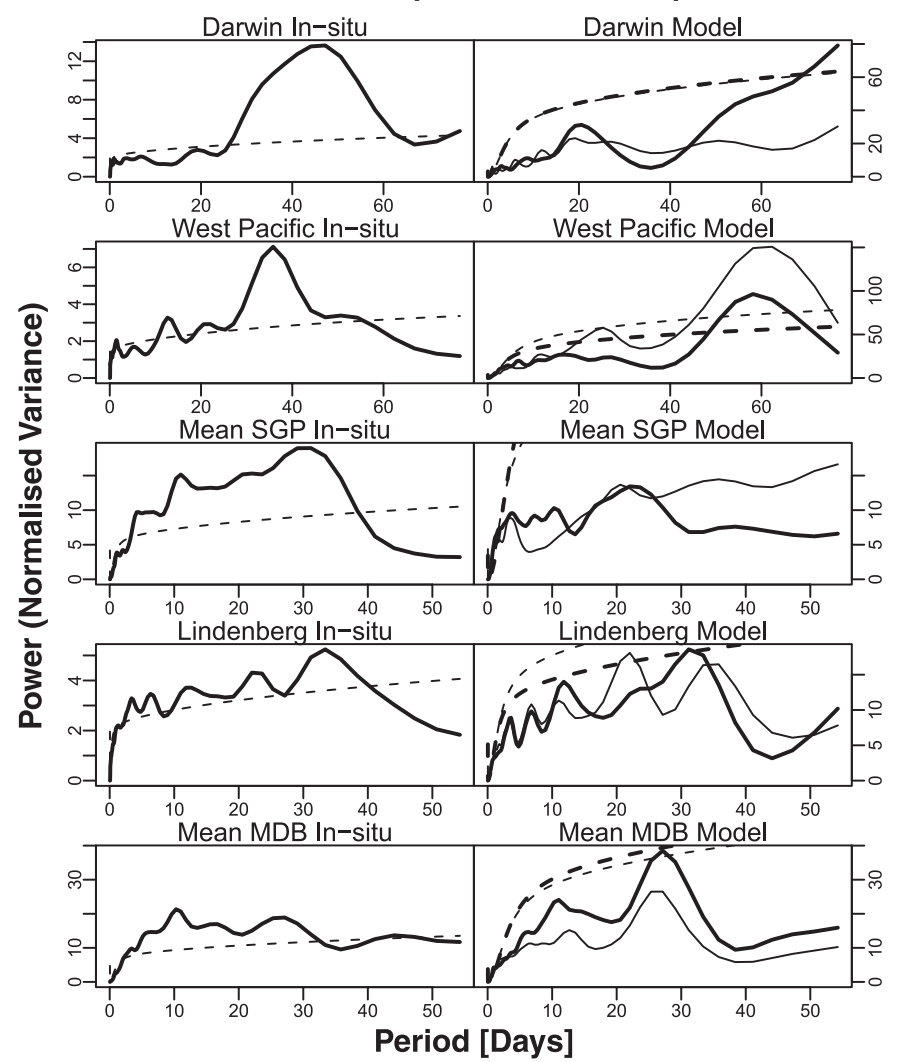

Fig. 18. The global wavelet power spectrum for precipitation for the in-situ data (left column) and the model (right) showing the longer periods. The ASSIM series is the heavy line on the right and the thin line the FORC series. The dashed lines are the corresponding line of statistical significance at the 5\% level. Note the differences in $y$-axis scale for between the in-situ and model plots for Darwin, the Western Pacific and Lindenberg.

high correlations with the in-situ monthly series. The lowest correlation occurs for the ASSIM series for the mean SGP where the model overestimates the rainfall for the first half of EOP3 and June 2003 but severely underestimates it for most of the rest of the period. The monthly precipitation for Equatorial Island and WPO is overestimated by the model in virtually every month although the general monthly variation is quite good for WPO which is reflected in the correlation coefficients. The overestimation for Equatorial Island is understandable given the mismatch in location; the rainfall on a high plateau should be less than the coastal rainfall but the result for WPO is interesting. The lack of land-sea inhomogeneity in the model should result in less convection than in the real world where differential surface heating and cooling should promote it. For
Lindenberg the model does not capture the monthly variation in the second half of the period although the annual amounts are quite well simulated.

The global wavelet power spectra are shown in Fig. 18. Note the differences in scale for Darwin, WPO and Lindenberg which are required to make the in-situ data peaks easier to see. Overall the model and in-situ spectra show peaks in similar frequency regions but the model has a tendency to overestimate their strength and underestimate their significance. Only the two mean in-situ data sets have spectra with power of similar strength to the model which may reflect their larger scale spatial nature. In all locations the two model time series show distinctly different behaviour to each other indicating that the precipitation fields in the ASSIM and FORC series are actually quite 


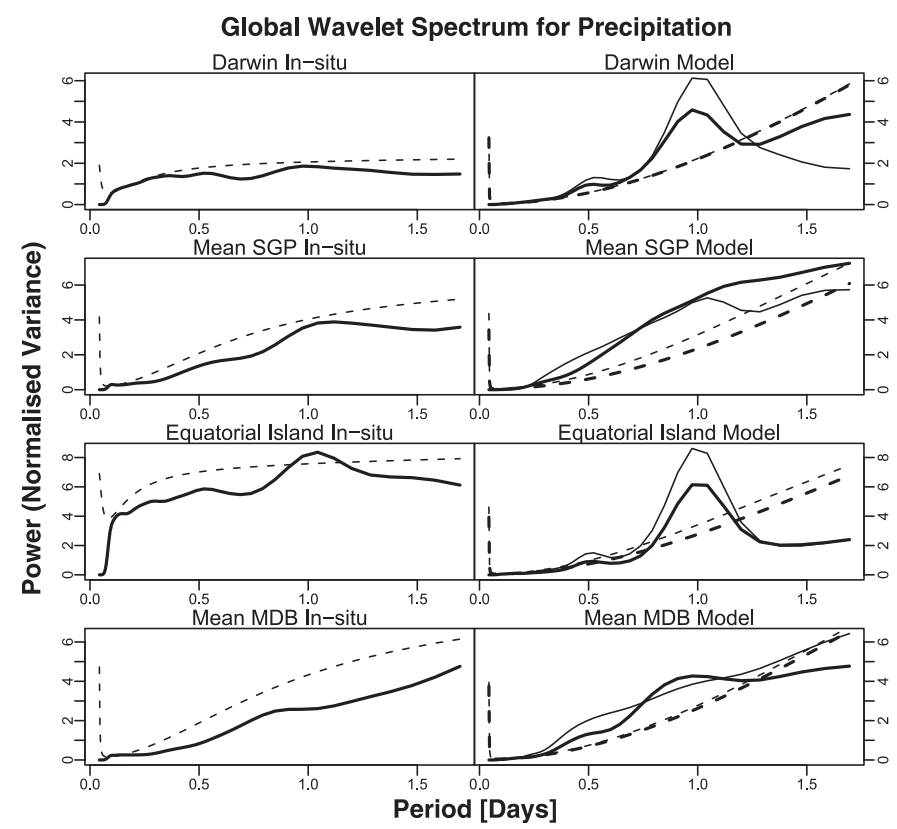

Fig. 19. The short period section of the global wavelet power spectrum for precipitation for the insitu data (left column) and the model (right). The ASSIM series is the heavy line on the right and the thin line the FORC series. The dashed lines are the corresponding line of statistical significance at the $5 \%$ level.

different fields. As shown in Fig. 18, the Darwin and Western Pacific observational data both have a significant (but weak) peak in the $40-$ 50 day period range which corresponds to the period of the Madden-Julian Oscillation (Madden and Julian 1994) but which is totally absent from the model results. For the midlatitude sites shown in Fig. 18 the model does not exhibit any statistically significant peaks. For the Mean SGP location the ASSIM series does reproduce some of the peaks out to periods of about 20 days but totally misses the peak at around 30 days. The corresponding FORC plot is very different to both the ASSIM and in-situ results. In the Lindenberg case both model spectra reproduce the in-situ peaks up to periods of about 40 days although the ASSIM series misses the peak at around 22 days which is present in the FORC spectrum. For the MDB both model series have too much strength in the peak centred around 27 days and the FORC spectrum lacks strength at lower periods where the ASSIM spectrum looks very similar to the in-situ pattern.

In the diurnal range spectral power plots shown in Fig. 19 the in-situ results have only the diurnal peak at Equatorial Island which is significant in direct contrast with the model results. While the model spectra generally reproduce the general shape of the in-situ spectra it clearly tends to overestimate the strength at the diurnal and semi-diurnal period range.

Figure 20 shows the annual mean diurnal variation of precipitation over the EOP3. All of the observational plots show significant discontinuities reflecting the intermittent nature of precipitation. The model, in contrast, has much smoother variations. Both Darwin and Lindenberg exhibit essentially flat behaviour which is consistent with smoothing out the observational data. The model is about 2 hours early for Equatorial Island but the overall structure is good. The ASSIM series is better than the FORC series for the mean SGP and MDB where there are significant differences between the two model data sets. The model does very well for WPO.

\section{Discussion}

It is a common to assume that data assimilation generated analysis are a reasonable proxy 


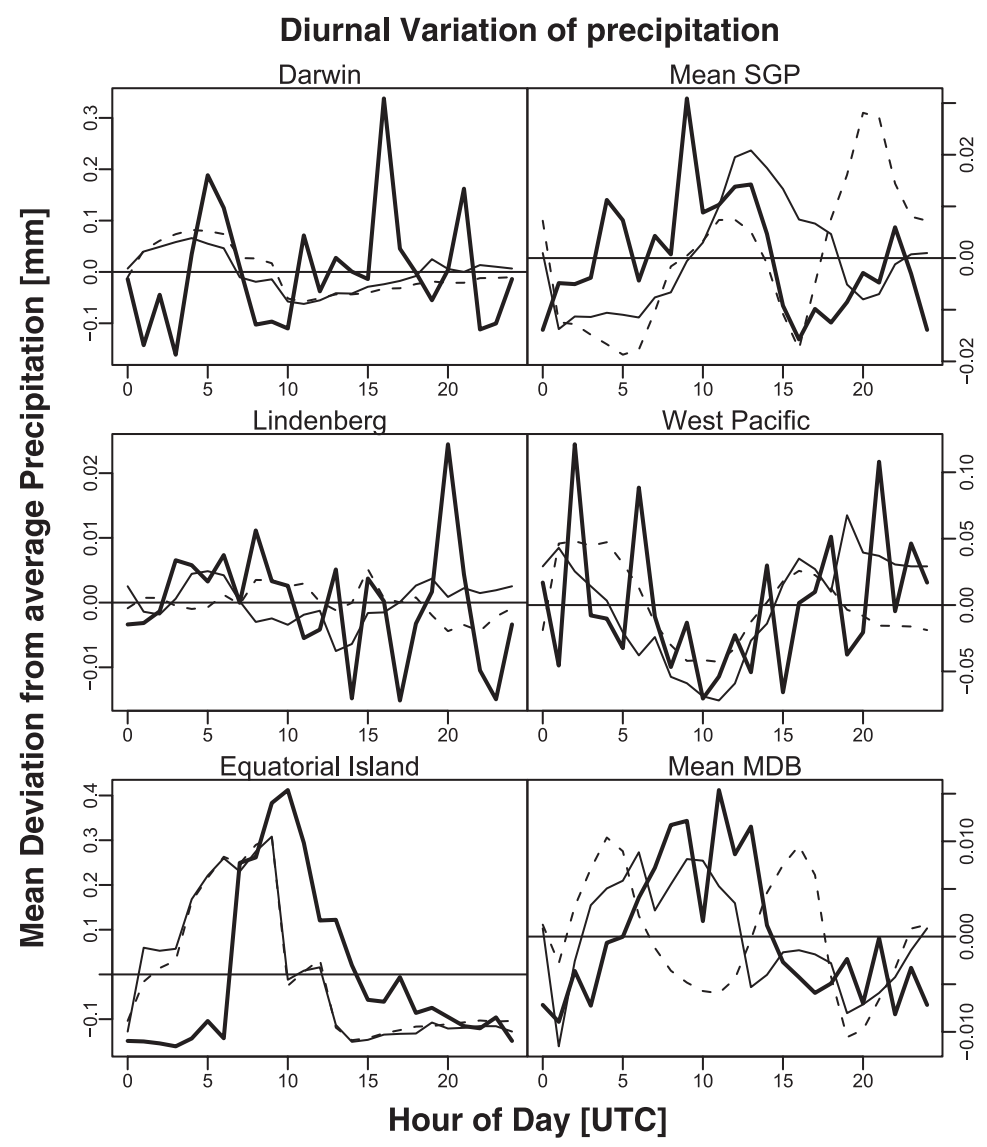

Fig. 20. The annual mean diurnal variation of precipitation for the MOLTS sites. The thick solid curves are the in-situ data, the thin lines the ASSIM and the dashed line the FORC results from the binning method described in the text.

for reality. When comparing such analyses with single location data there is no real reason to expect this to be true; the data at a geographical point is very different to model data which is valid at large grid scales. Variables such as surface pressure and temperature seem to be quite representative at model grid scales but wind and precipitation certainly are not. The limitations of the assimilation scheme also need to be accounted for; for example, water vapor is very poorly characterized due to its very strong scale dependence and a sparsity of definitive data. This problem will be a feature in all assimilation schemes to some extent because the moisture fields vary substantially at subgrid scales that are not representable in the scheme. The sophistication of the model physics also needs to be evaluated; all models have problems with the parameterization of convec- tion and cloud in general, and this will be reflected in their description of the moisture field which will in turn have impacts on the analysis. Model spin up (or down) can play a role; in the Bureau's assimilation scheme the independent univariate moisture analysis can produce thermodynamic fields which are not in a balance with the model physics almost ensuring large changes in the early part of a forecast although some fields will show longer scale systematic errors. These spin up errors are unlikely to be random; they will have variations dependent on regional areas and meteorological conditions. They are much more likely to appear in data sparse regions where there is insufficient information to correctly characterize the true atmospheric state and the introduction of new data will cause 'jumps' as the assimilation system applies large corrections. In many 
cases these jumps will not be restricted to moisture variables.

Validation of the model with the Equatorial Island data showed differences which could be attributed to the stark differences in the situation of the points; the in-situ data corresponds to a mountain plateau and the model to a coastal site. Hence the smaller model annual mean diurnal variation in temperature is consistent but the agreement in diurnal signal for humidity is more surprising. The differences between the model and the in-situ sites for WPO are reasonably consistent with the lack of topography in the model. The site which fared worst in the comparison process is Darwin. This is not surprising given the dominant role of convection in Darwin's weather and the overall problems with convective parameterization schemes. The annual mean diurnal variations diagnosed in this study are certainly too simplistic; the seasons in Darwin are dramatically different and should obviously be treated separately. However, they do point to some interesting areas which merit further investigation, particularly the severe phase errors in variables such as humidity and wind speed. The model also shows a strong sensitivity to the SST which, because it is kept constant between weekly updates, suppresses the diurnal variation at some locations near the ocean. Depending on the model's horizontal resolution the influence of constant SST's may affect regions in the model even further away. Comparisons of global wavelet power spectra have shown that for all variables except precipitation the model is generally very similar in its description of the dominant modes of variability with some notable exceptions such as the screen level humidity for Lindenberg and the WPO. The largest discrepancies tend to be at the longer periods of variability where the model often shows a tendency to push the power to longer periods. One aspect of the wavelet transform approach which was not explored here is the ability to diagnose the time series of the variations in the strength of different frequencies of variation (e.g., diurnal) which might allow a better analysis of the causes of the differences between the model and in-situ data.

Overall, the results of this study have shown that the time series for the model and the in- situ data are very similar for surface pressure, and grow progressively more independent in the comparisons for screen level humidity, temperature and wind speed at $10 \mathrm{~m}$. The time series for precipitation show little similarity. The limitations of considering just single MOLTS points has been highlighted by this study; the next version of the Bureau's model will produce MOLTS data for the four surrounding points as well as full grid data at a lower temporal resolution.

The biggest advantage of the CEOP data set is its use of standard formats and consistent quality control so that it should be a simple matter to apply the programs developed for this study to the complete set of relevant insitu sites as well as the other contributing models. The range of variables also needs to be expanded to address the main concerns of the CEOP program; namely the energy and hydrology budgets. These extensions are the subject of work in progress.

\section{Acknowledgements}

The ARM data were obtained from the Atmospheric Radiation Measurement (ARM) Program sponsored by the U.S. department of Energy, Office of Science, Office of Biological and Environmental Research, Environmental Sciences Division.

The data for the Western Pacific Ocean site was collected and is provided by the Institute of Observational Research for Global Change (IORGC), Japan Agency for Marine-Earth Science and Technology (JAMSTEC).

The original data for Equatorial Island was collected and is provided within the framework of the research collaboration between Frontier Observational Research System for Global Change (FORSGC), Japan Marine Science and Technology Center (JAMSTEC) and Radio Science Center for Space and Atmosphere (RASC), Kyoto University, financially supported by the Japanese Ministry of Education, Science and Culture.

The Lindenberg data was supplied by Deutscher Wetterdienst (DWD)-Meteorologisches Observatorium Lindberg/Richard Assmann Observatorium.

I would like to thank Peter Steinle for useful discussions on assimilation schemes, Jeff Kepert, and Bill Bourke for useful suggestions on 
improving the paper, Noel Davidson for sharing his tropical meteorology knowledge, and Alan Seed for discussions on the wavelet spectra, as well as the other contributors to the CEOP project.

\section{References}

Beyrich, F., H.-J. Herzog, and J. Neisser, 2002: The LITFASS project of DWD and the LITFASS-98 experiment; The project strategy and the experimental setup. Theor. Appl. Climatol., 73, 3-18.

Charnock, H., 1955: Wind stress on a water surface. Quart. J. Roy. Meteor. Soc., 81, 639-640.

Dai, A. and J. Wang, 1999: Diurnal and semidiurnal tides in global surface pressure fields. J. Atmos. Sci., 56, 3874-3891.

Fels, S.B. and M.D. Schwarzkopf, 1975: The simplified exchange approximation: A new method for radiative transfer calculations. J. Atmos. Sci., 32, 1475-1488.

Hummel, J.R. and R.A. Reck, 1979: A global surface albedo model. J. Appl. Meteor., 18, 239253.

Kubota, H., R. Shirooka, T. Ushiyama, J. Chen, T. Chuda, S. Iwasaki, and K. Takeuchi, 2002: Seasonal variability observed at Palau over western Pacific and water vapor variation observed prior to the large-scale convective activity, Western Pacific Geophysical Meeting Abstracts, AGU, WP8-WP9.

Lacis, A.A. and J.E. Hansen, 1974: A parameterization for the absorption of solar radiation in the earth's atmosphere. J. Atmos. Sci., 31, 118133.

Lemus, L., L. Rikus, and C.M.R. Platt, 1997: Global Cloud Liquid Water Path Simulations, J. Climate, 10, 52-64.

Louis, J.-F., 1979: A parametric model of vertical eddy fluxes in the atmosphere. Bound. Layer Meteor., 17, 187-202.

Madden, R.A. and P.R. Julian, 1994: Observations of the 40-50 day tropical oscillation: a review. Mon. Wea. Rev., 122, 814-837.

Manabe, S. and J.L. Holloway, 1975: The seasonal variation of the hydrologic cycle as simulated by a global model of the atmosphere. J. Geophys. Res., 80, 1617-1649.

Murata, F., M.D. Yamanaka, M. Fujiwara, S.-Y. Ogino, H. Hashiguchi, S. Fukao, M. Kudsy, T. Sribimwati, S.W.B. Harijono, and E. Kelana, 2002: Relationship between wind and precipitation observed with a UHF radar, GPS rawinsonde and surface meteorological instruments at Kototabang, West Sumatra during
September-October 1998. J. Meteor. Soc. Japan, 80, 347-360.

Neisser, J., W. Adam, F. Beyrich, U. Leiterer, and H. Steinhagen, 2002: Atmospheric boundary layer monitoring at the Meteorological Observatory Lindenberg as a part of the "Lindenberg Column"; Facilities and selected results. Meteorol. Z. (N.F.), 11, 241-253.

Payne, R.E., 1972: Albedo of the sea surface. J. Atmos. Sci., 29, 959-970.

Rew, R.K. and G.P. Davis, 1990: The Unidata netCDF: Software for Scientific Data Access, Sixth International Conference on Interactive Information and Processing Systems for Meteorology, Oceanography, and Hydrology, Anaheim, California, American Meteorology Society, pp 33-40, February 1990.

Rikus, L., 1991: The role of clouds in global climate modelling. BMRC Report No. 25, Bureau of Meteorology Research Centre, Melbourne, Australia, $37 \mathrm{pp}$.

Schwarzkopf, M.D. and S.B. Fels, 1991: The simplified exchange method revisited: An accurate, rapid method for computation of infrared cooling rates and fluxes. J. Geophys. Res., 96, 9075-9096.

Seaman, R., W. Bourke, P. Steinle, T. Hart, G. Embery, and M. Naughton, 1995: Evolution of the Bureau of Meteorology's Global Assimilation and Prediction system. Part 1: Analysis and initialisation. Aust. Met. Mag., 44, 19-40.

Siriwardena, L., F. Chiew, H. Richter, and A. Western, 2003: Preparation of a climate data set for the Murrumbidgee River Catchment for land surface modelling experiments. Cooperative Research Centre for Catchment Hydrology working document 03/1, Cooperative Research Centre for Catchment Hydrology, Clayton, Victoria, Australia.

Smith, N.R., 1995: The BMRC ocean thermal analysis system. Aust. Met. Mag., 44, 93-110.

Tiedtke, M., 1989: A Comprehensive mass flux scheme for cumulus parameterization in large scale models. Month. Wea. Rev., 117, 17991801.

Torrence, C. and G.P. Compo, 1998: A Practical Guide to Wavelet Analysis. Bull. Amer. Meteor. Soc., 79, 61-78.

Yang, K., T. Koike, K. Tamagawa, and P. Koudelova, 2005: Model Uncertainties Correlated with Spatial Variability of Prognostic Variables. Contrib. to The $85^{\text {th }}$ AMS Annual Meeting, San Diego, USA, 9-13 January, 2005.

Yang, G.-Y. and J. Slingo, 2001: The diurnal cycle in the tropics. Month. Wea. Rev., 129, 784-801. 